

$$
\text { L }
$$


Design of Fast Connected Components Hardware

by

Xue Dong Yang

Technical Report No. 353

Robotics Report No. 143

March, 1988

\author{
New York University \\ Dept. of Computer Science \\ Courant Institute of Mathematical Sciences \\ 251 Mercer Street \\ New York, New York 10012
}

Work on this paper has been supported by Office of Naval Research Grant N00014-87-K. 0129 National Science Foundation CER Grant DCR-83-20085, National Science Foundation Grant subcontract CMU-406349-55586, and by grants from the Digital Equipment Corporation and the IBM Corporation. 



\title{
Design of Fast Connected Components Hardware
}

\author{
Xue Dong Yang \\ Computer Science Department \\ Courant Institute of Mathematical Sciences \\ New York University \\ 251 Mercer Street \\ New York, NY, 10012
}

\begin{abstract}
The intensive use of the connected components algorithm in image analysis and robot vision calls for a very fast implementation of such algorithm suitable for realtime applications. This paper presents a hardware design implementing the algorithm due to Schwartz, Sharir, and Siegel[8]. A prototype board, without using special VLSI chips, had been constructed in Robotics Research Lab of Courant Institute. It can compute the connected components of a $512 \times 512$ binary image in few video frame times (about $300 \mathrm{~ms}$ ). A real-time version (video speed) in VLSI is proposed.
\end{abstract}

\section{Introduction}

The labeling of connected components of a binary image is a fundamental problem in image analysis. An early method was developed by Rosenfeld and Pfaltz [7] in 1966; it uses a pair of arrays, one containing the current region label and the other containing its smallest equivalent label. This algorithm processes the image from top to bottom to compute label equivalences, storing the result in the arrays. A second pass reassigns each label to its smallest equivalent label. Lumia, Shapiro, and Zuniga [3] improved the previous method by using a very short equivalence table, which needs to cover only one line. Schwartz, Sharir, and Siegel [8] present a method which uses bracket marking to associate equivalent groups. This method enables one to compute the component numbers for each pixel on the fly, by using an relative small auxiliary 'bracket table'. More interestingly, this algorithm uses only simple form of pushdown-stacks, making high-speed hardware realization possible. In addition to the above mentioned sequential algorithms, Shiloach and Vishkin [9] present a logarithmic-time connected components algorithm known for massively parallel computing systems (e.g. 1 processor per pixel) connected in shuffleexchange or other similar pattern.

In this paper, we present a hardware implementation of the algorithm due to Schwartz, Sharir, and Siegel[8]. In the following sections, data structures and algorithm are described in detail. Then the hardware design realizing the algorithm is presented. A prototype Connected Components Board, which does not use any specially designed VLSI chips, has been constructed at Robotics Research Lab. of Courant Institute, NYU. It can label the connected components of a $512 \times 512$ binary image in just a few video-frame times (about $300 \mathrm{~ms}$ ). Real-time computation (i.e. video speed) can be achieved without significant redesigning by pipelining the two major computing components of the board. For a connected components algorithm to be suitable for intensive use in real-time image processing and robot vision 
applications, it must allow for a very fast implementation. In the last section we discuss this enhancement and outline a possible VLSI implementation of the algorithm.

\section{The Algorithm and Its Implementation}

The algorithm that we have implemented was first presented in [8]. For the sake of completeness, a description of the algorithm, and the details which are relevant to its hardware implementation are given below. First, we review key definitions as follows:

Definltion 1: Let $R_{p}$ be an image row, $(1 \leq p \leq m)$.

(a) $A$ run in $R_{p}$ is a sequence of 1-pixels (i.e. pixels with gray value 1) of $R_{p}$ bounded on both sides by 0-pixel (For simplicity, we add two additional 0-pixels to the left and right-end of each row of the image, respectively).

(b) The lower semi-image $I_{p}$ consists of the union of all rows $R_{j}, p \leq j \leq m$.

(c) $G_{p}$ is defined to be the partition of the set of runs in $R_{p}$, for which two runs are in the same partition group $g \in G_{p}$, iff they belong to the same connected component of the lower semi-image $I_{p}$.

The algorithm consists of two passes. Pass 1 sweeps through the rows from bottom to top, during which the groups in $G_{p}$ are calculated inductively from the knowledge of $R_{p}+1$ by a simple updating rule. Pass 2 sweeps from top to bottom to assign component numbers to each pixel and outputs this symbolic image.

In each row, runs belonging to the same group are associated by a simple mechanism, called bracket marking, as follows:

Definition 2: We define four symbols '[', ']', '[]', and '] ['.

(a) If $r$ is the leftmost (resp. rightmost) run in its group $g$, it is assigned marking l (resp. J).

(b) If $r$ is both leftmost and rightmost run in $g$. i.e. 8 consists of a single run $r$, then $r$ is assigned the marking [].

(c) If $r$ lies between the leftmost and rightmost run in its group, it is assigned the marking $]$.

An example is given in Fig 2.1, where a bracket marking is shown for seven runs in $R_{p}$ numbered from right to left. These seven runs are divided into three groups - $(7,4,3,1),(6,5)$, and $(2)$. It is shown in [8] that the bracket sequence associated with row $R_{p}$ is properly nested, i.e. each right bracket is matched to an associated left bracket and vice versa. Hence, the groups into which we divided the set of all runs in $R_{p}$ can be reconstructed from the bracket sequence associated with $R_{p}$.

\subsection{Pass 1}

The goal of pass 1 is to calculate a bracket marking for each row by sweeping from bottom to top, and to store it in a bracket table (to be accessed in pass 2 ). For this we want a rule telling us how to calculate the bracket marking for the row $R_{p}$ 


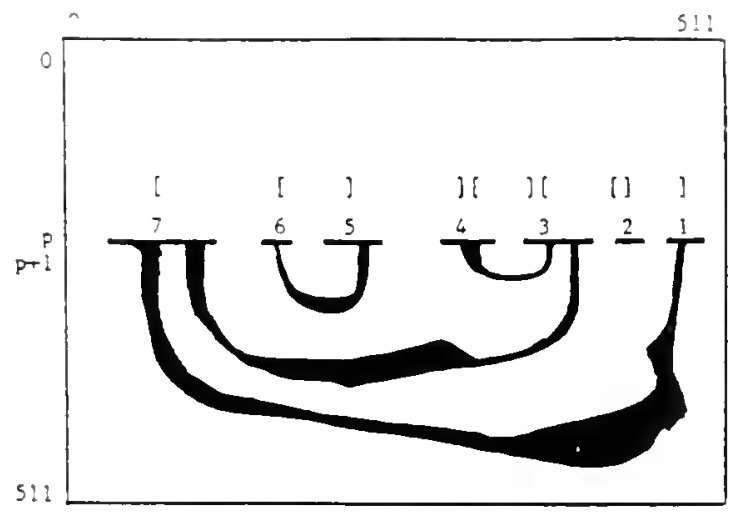

Figure 2.1 An example of bracket marking assignment

given the same information for $R_{p}+1$. The key observation is that any two runs in row $R_{p}$ will be in a group $g \in G_{p}$ iff there are some runs in $g^{\prime} \in G_{p+1}$ whose index set has a nonempty intersection with the index sets of the two runs in $R_{p}$. Intuitively, those runs in row $R_{p}$ which are linked together by a chain in the semi-image $I_{p}$ belong to the same group. The algorithm scans two rows, $R_{p}$ and $R_{p}+1$, simultaneously from right to left. Two pushdown stacks, stack1, and stack2, are used in pass 1. Stackl is used for tracing the group relationship represented by the bracket sequence in $R_{p}+1$ as following:

(a) If a run with ']' is encountered, we push a unmarked symbol onto stack1;

(b) If a run with '][' is encountered, we neither push nor pop;

(c) If a run with ' $[$ ' is encountered, we pop the top entry off stack 1 at the end of this Iun;

(d) If a run with '[]' is encountered, we push an unmarked symbol onto stackl at the beginning of the run, and pop stackl at the end of the run.

When we are within a run in $R_{p}+1$ during scan, if we encounter a run in $R_{p}$, then we mark the symbol on the top of stackl, which indicates that the current group in $R_{p+1}$ has touched some run in $R_{p}$. When a run in $R_{p}$ is encountered and we are within a run in $R_{p}+1$ with a '][' or '[' marking, we look at the top entry of stackl to see if it is marked, i.e. to see if the current group in $R_{p+1}$ already touched previously some run in $R_{p}$. If so, the current encountered run in $R_{p}$ is linked to some run to its right in the same row through a path below $R_{p}$.

Stack2 is used for maintaining the group information about the row $R_{p}$ during the scan. Assume we number each run in row $R_{p}$ from right to left starting from 1 , as an example shown in Fig. 2.1. Each entry in stack2 is a pair of integers, representing the right-most and left-most runs of each group known to the point we have reached during scan. This group boundary information is updated according to the new knowledge we gain about each group as the scan continues. Given the way that we scan a row from right to left, the connectivity of runs in row $R_{p}$ through links in the semi-image $I_{p+1}$ can be classified into three basic cases as follows (refer to Fig. 2.2):

(a) Several runs may be linked by a simple chain;

(b) A cluster of links (or arcs) may be bound together on its right end by a single run in row $R_{p}$;

(c) A cluster of links (or arcs) may be tied together on its left end by a single run in row $R_{p}$. 


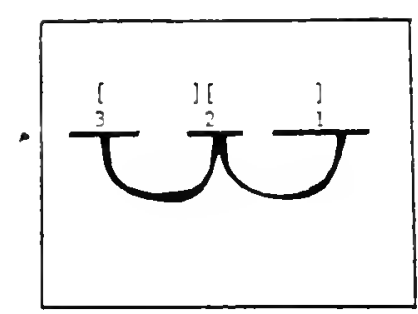

(a)

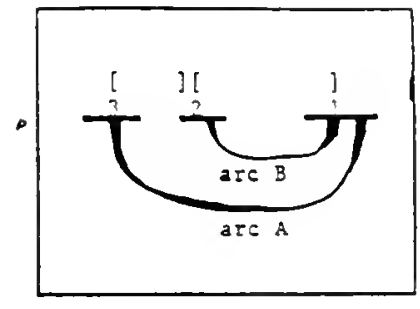

(b)

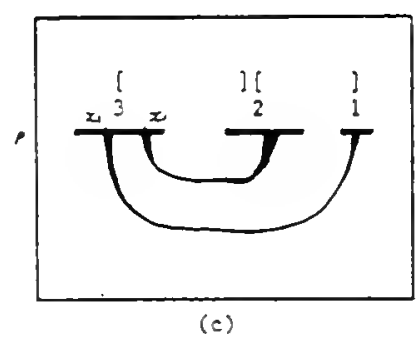

Figure 2.2 Basic cases of connectivity in semi-image $I_{p}$ :

(a) simple chain; (b) a cluster of links is bound at right end;

(c) a cluster links is bound at left end.

The general case can be decomposed recursively into these three elementary cases by recursively considering a subset of consecutive runs in a group as a "single run". For example in Fig. 2.1, if we consider runs 4 and 3 as a "single run" denoted by $4-3$, the connectivity between $7,4-3$, and 1 falls into the elementary case (c). Our algorithm handles the connectivity problem from inner level to outer level recursively. Thus, at any time, we only have to deal with one of the three elementary cases defined above; however a run could be a simple run or a virtual "single run".

\subsubsection{Manipulation of Stack 2 Information}

The condition for beginning a new group can be easily checked locally. When we encounter a new run in row $R_{p}$, we check if it makes contact with any run in row $R_{p}+1$. If it does not, we know that it is the start of a new group containing one run only. On the other hand, if it does make contact with some runs in $R_{p+1}$ the right most of which has a ']' marking, we assume temporarily that a new group starts. (We say 'temporarily' here since initially no link connects the current run in $R_{p}$ to a run to its right on same row. However this new run might link to some run to its right indirectly through several arcs. For example in Fig. 2.2(c), run 2 links indirectly to run 1 through two arcs.)

\section{Case (a)}

In case (a) of Fig. 2.2 , we have entry $[1,1]$ on stack 2 after reaching run 1 , which means the left and right bound of the current group are 1 and 1 respectively. The left bound expands to 2 after run 2 , resulting in the top stack entry $[1,1]$ being updated to $[2,1]$. It is then updated to $[3,1]$ when run 3 is reached, after which it is popped off stack2 since a group is finished. So in case (a), the group always expands its left bound. 


\section{Case (b)}

The situation in case (b) of Fig. 2.2 is slightly more complicated. We have $[1,1]$ on stack2 after run 1. (Remember that we have two marked entries on stackl after run 1 in row $R_{p}$ since it touched two runs in row $R_{p+1}$ both of which have ']' brackets.)

After run 2, the top entry on stack 2 becomes $[2,1]$ and the top entry on stackl was popped. At this point we do not know whether or not to pop the top entry on stack2. While it is possible, using only local information to determine the start of a new group, this is not the case for determining the end of the current group. The fact that a run has no more arcs linking it leftwards does not necessarily mean that the end of this group has been reached. (Note that run 2 is further linked to run 3 through a indirect path.)

To handle this situation, we introduce an auxiliary field in stackl's entry to indicate if the arc associated with a ']' marking in that entry is the outermost (or lowest) arc. For example, we say arc $A$ is the lowest among the cluster of arcs bounded by run 1 in row $R_{p}$ in (b) of Fig.2.2. The auxiliary bit can be easily set as following. During a run in row $R_{p}$, if one or more entries have to be pushed onto stackl, the auxiliary field in the first pushed entry is set to TRUE and the auxiliary fields in all other entries are set to FALSE. If the auxiliary field in the entry just popped off stackl is FALSE, we know the arc (or link) just ended is not the outermost one (lowest), i.e. there is an arc belonging to the same group enclosing this finished arc, so we don't pop the entry off stack 2 because we don't know if the current group in row $R_{p}$ is finished yet. After reaching run 3 in row $R_{p}$, the left bound of current group is expanded to 3 , and the top entry on stack 2 is changed from $[2,1]$ to $[3,1]$. When the entry on stackl is popped off, we see that the auxiliary field is TRUE. So, we can confidently pop the top entry on stack 2 off this time because we know that there is no further arc linking this group to any run to its left.

\section{Case (c)}

A situation symmetrical to case (b) occurs in case (c). After reaching run 1 , we have one entry, $[1,1]$, on stack 2 . Note that at the moment after reaching run 2 , it is impossible to know that this run is actually linked to run 1 through an indirect path. So, we have a second entry, [2,2], on top of stack2. After point $x_{1}$ in run 3 is reached, the top entry of stack2 is updated from $[2,2]$ to $[3,2]$. When we reach point $x_{2}$ we find that two arcs are bound together. So, the two top entries on stack2 are merged into one in such a way that the new left bound is the left bound of first entry on stack 2 and the new right bound is the right bound of the second entry of stack 2 , resulting a $[3,1]$ on stack 2 . These operations can be performed in two steps: (1) pop stack2; (2) modify the left bound of top entry by the left bound of just popped entry. In this example, we pop [3,2] off stackI first; then change the left field of top entry - $[1,1]$ to 3 and get $[3,1]$ as the new top on stackl.

\subsubsection{Bracket Table}

The bracket marking can be effectively encoded by a two-bit binary number as shown in Table 2.1. The bracket information calculated during pass 1 is stored in a memory of $512 \times 256 \times 2$ bits, called the brackets table. This table is indexed by two 
Table 2.1 Bracket Marking Encode Definitions

\begin{tabular}{|c|c|c|}
\hline Bracket Marking & Left Bit & Right Bit \\
\hline[] & 0 & 0 \\
\hline[ & 0 & 1 \\
\hline$]$ & 1 & 0 \\
\hline$][$ & 1 & 1 \\
\hline
\end{tabular}

row counters, row_count 1 and row_count2, and two column counters, run_countl and run_count2. Row_countl and run_countl are combined to access brackets in row $R_{p}$, and row_count 2 and run_count 2 for those in row $R_{p+1}$. Each entry in the table has two 1-bit fields, both of which are initialized to zero. Run_counts are set to zero at the beginning of a row sweep and incremented by 1 every time a new run started.

Now we show how the bracket table can be updated efficiently as groups grow during scan. There are only two types of group-expanding operations: (a) the left boundary of the current group expands to include the current scanned run; (b) the two top most groups on stack 2 are merged together. To do each of these operations in constant time, we must be able to directly index correct columns in a row of the bracket table. The index of the current scanning run is provided by run_countl for row $R_{p}$ and run_count 2 for row $R_{p}+1$. The boundaries of all non-ended groups are maintained in stack 2 in a proper nested order with the current group on the top. Thus, the updating of bracket table's entry in every possible case can be done in constant time. By the end of pass 1 , we get a complete bracket marking for every run of the binary image stored in the bracket table.

\subsection{Pass 2}

In pass 1 we have only solved the connectivity problem for runs in each row that connect through some links below that row. However, the connectivity between those runs in a row which are linked together only through some arcs above that row can not be found in this bottom-to-top sweep. A second pass sweeping in the opposite direction is necessary to solve the connected components problem completely.

Pass 2 is much simpler than pass 1 . On each row, two sweeps are used. The first sweep scans the pixels in two consecutive rows, $R_{p-1}$ and $R_{p}$, simultaneously from right to left. The second sweep scans row $R_{p}$ from left to right only. We still use two stacks, stack3 and stack4, but they play slightly different roles to the two stacks used in pass 1. Stack 3 is used to trace the group information in row $R_{p}$, and stack 4 saves the component number assigned to each group in row $R_{p}$. A circular queue is used for temporarily storing component numbers corresponding to each run in row $R_{p-1}$ with the right-most run at the head of the queue.

The first sweep induces the component number already assigned to runs in row $R_{p-1}$ to the runs in row $R_{p}$ to which they are connected, and allocates a new component number to each new group emerged in row $R_{p}$.

When a run with ']' bracket is encountered in row $R_{p}$, which means the start of a 
new group has been found, we push a NULL onto stack3. On the other hand, when a run is encountered in row $R_{p}-1$, we remove the entry at the tail of the queue which contains the component number corresponding to this run. Then if the current group of row $R_{p}$ connects with any run in row $R_{p-1}$ and the top of stack 3 is still NULL, we replace the top of the stack3 by the entry most recently removed from the queue. When a run with a '[' bracket is encountered in row $R_{p}$, which indicates the current group has ended, we pop the top entry from stack3. If this popped element is not NULL, it is pushed onto stack4; otherwise, a new component number is allocated and is pushed onto stack4. At the end of sweep 1, we have the component numbers for each groups of row $R_{p}$ in stack 4 with the last ended group's on the top.

The second sweep scans row $R_{p}$ only, from left to right. When a run with '[' bracket is encountered, we pop its component number from stack 4 and push it onto stack3. The component number of a group on stack 3 is popped off at the end of the group's right-most run which must have a ']' bracket. Each pixel scanned is checked to see if it is white. If it is, then the background component number is output. Otherwise it is within a run, so we output the content on the top of stack 3 which is the component number of current group.

The full algorithm written in C Programming Language is given in Appendix A.

\section{Hardware Design}

In section 2 , we have shown that all operations involved in the computation are

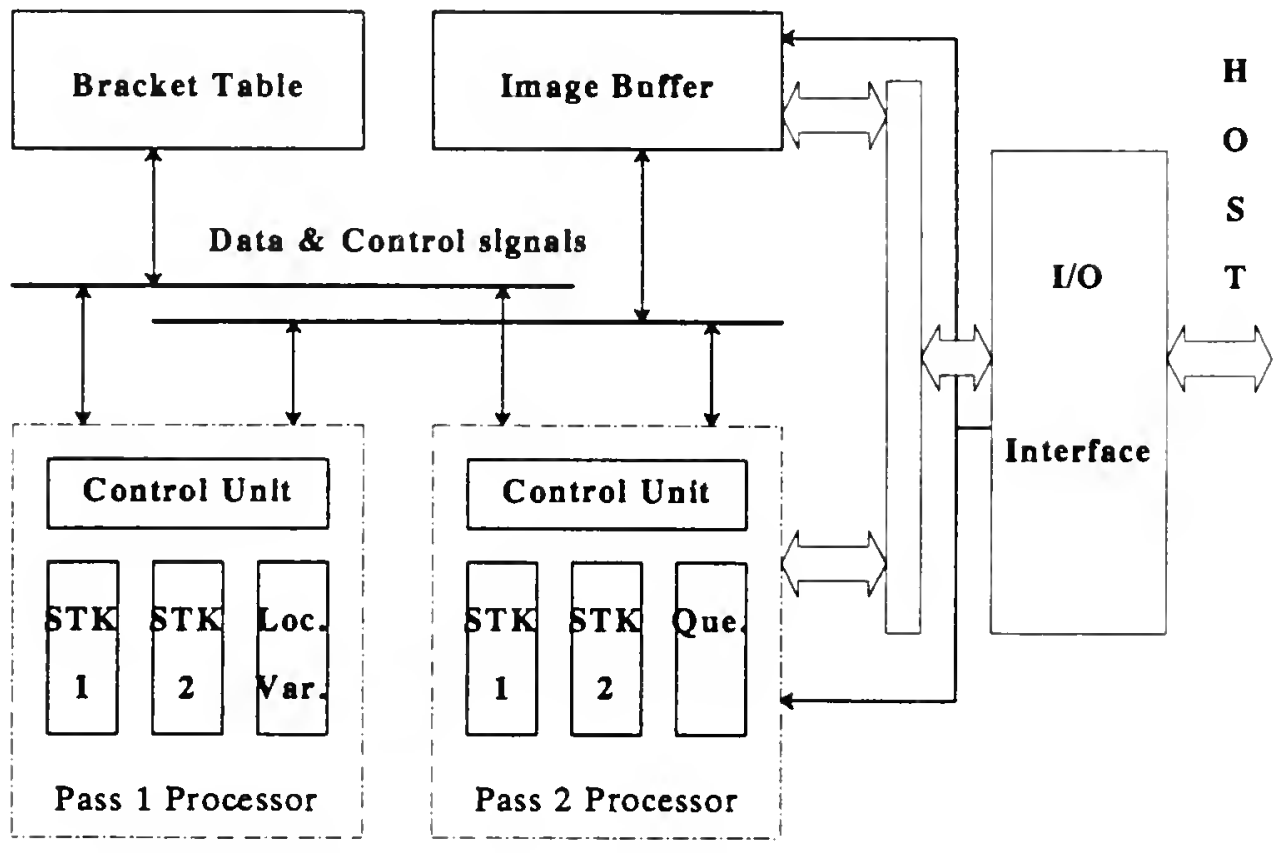

Figure 3.1 Block Diagram of Connected Component Board 
simple and can be classified as follows:

(a) Simple variable assignment (register transfer).

(b) Indexed memory access (e.g. bracket table read/write).

(c) Push stack, pop stack, or modify the top of stack.

(d) Insert queue and delete queue.

It should, and does indeed, have a straightforward implementation in hardware.

The goal of the first prototype connected components board designed was to implement the algorithm described in section 2 in standard MSI or LSI chips without involving special VLSI chip design, and the computation on a $512 \times 512$ binary image can be accomplished in few video-frame times. Real-time implementation and VLSI design are discussed in the last section of this paper.

The design is carried out in a systematic way. We start by specifying modular units and connections between them. Then the data structure and functions performed in each module are precisely described by a number of processes written in a special microparallel system hardware design language (see Appendix B). Processes are then mapped into circuits by several regular rules. Our explanation concentrates on the architecture of the hardware. The low level schematic design can be found in [10].

The block diagram of the connected component board is shown in Fig. 3.1. There are five major components:

(1) Pass 1 processor.

(2) Pass 2 processor.

(3) Image buffer.

(4) Bracket table.

(5) Input/Output interface.

In following, we examine each unit by describing the main data structure of the component along with its control structure.

\subsection{Control Units}

The computation of the algorithm is carried out by Pass 1 Processor and Pass 2 Processor. Both processors have very similar both data and control structures.

As can been seen in Fig. 3.1, Pass 1 processor contains a control unit, two stacks, and some local variables. The control unit coordinates the register transfers, stack operations, image buffer and bracket table accessings, according to the algorithm by issuing pulses to each component in an appropriate order. The control unit is implemented by a typical time sequential circuit or so called finite state machine. The structure of a finite state machine is given in Fig. 3.2 and its principle is explained in many digital logic design books, e.g. [11]. The output signals are control signals to each data components. The logical structure of control flow in the control unit of pass 1 is depicted in Fig.3.3.

There are two nested loops in the diagram, with the outer one iterating on rows and the inner one on columns. There are number of modules, so called processes, in Fig. 3.3. All processes are self-timed. Within each process some control signals are issued to data components to carry out manipulations. For example, when Pass1_Initialization process is activated, it sends control signals to set initial state for 


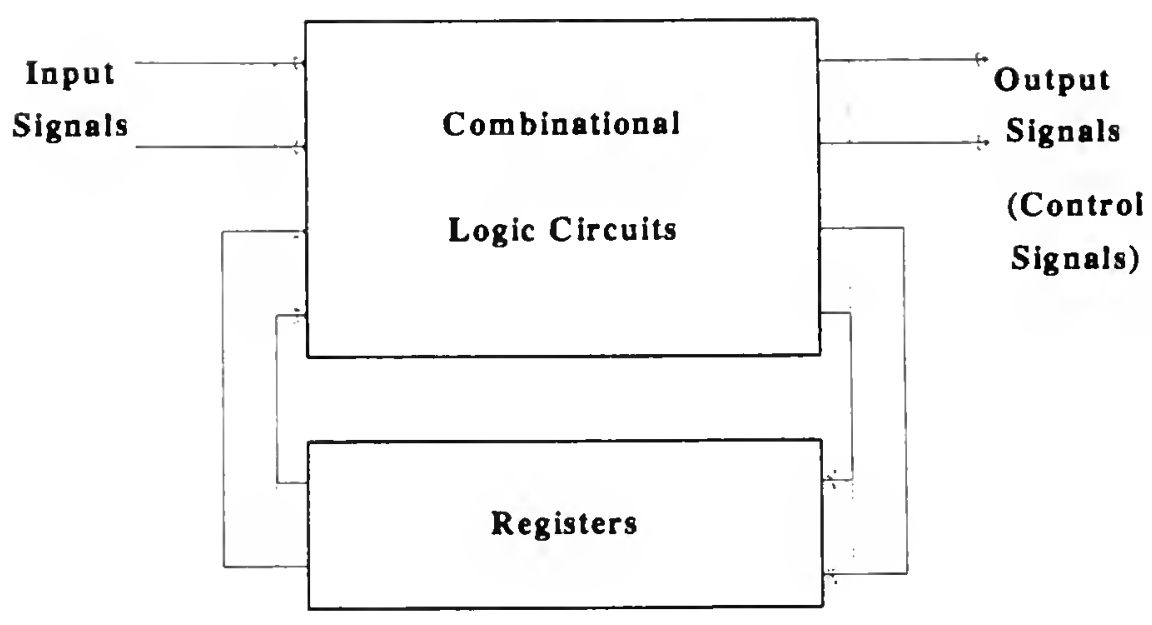

Figure 3.2 Block diagram of finite state machine

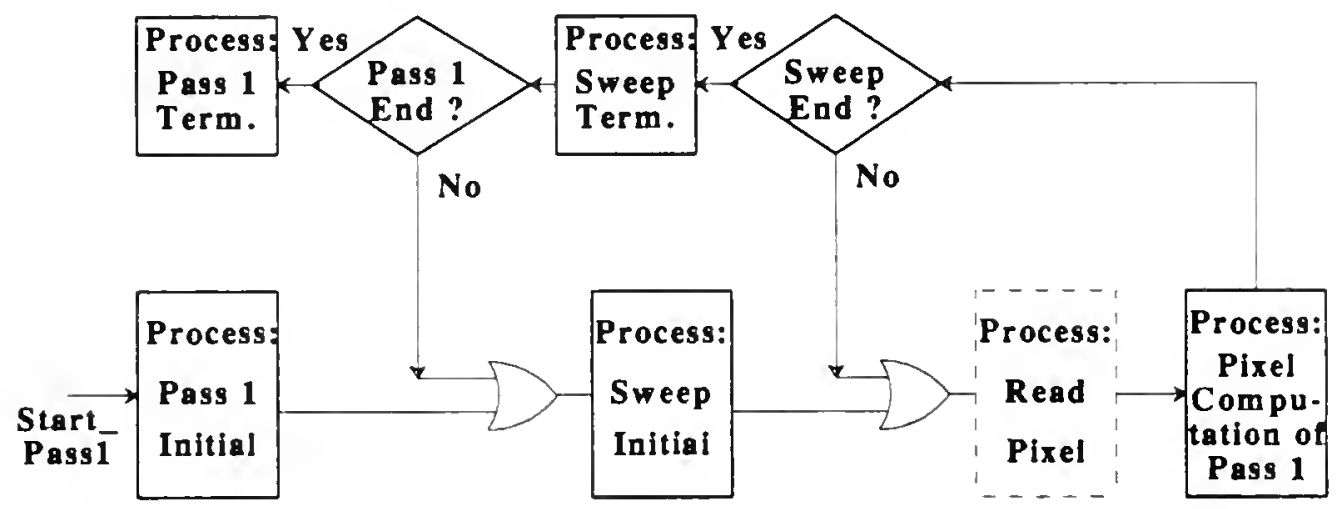

Figure 3.3 The control unit of Pass 1 processor

pass 1 in various data components. The procedural descriptions of those processes can be found in Appendix B.

The control unit of the Pass 2 Processor has a similar structure as the Pass 1 Processor. Fig. 3.4 shows the logical control structure of the Pass 2 control unit. It contains three loops. The outer loop iterates on rows. The two inner loops are concatenated in serial. The first inner loop iterates on columns from right to left in a row; the second loop iterates in the opposite direction.

\subsection{Data Components}

\subsubsection{Pushdown Stacks}

The most important data structure here is the pushdown stack. The depth of each stack used is 256. A bi-directional shift-register is seems the best element for implementing a pushdown stack. Since we couldn't find commercial shift-registers of such length, each stack in our design is constructed painfully from a piece of 


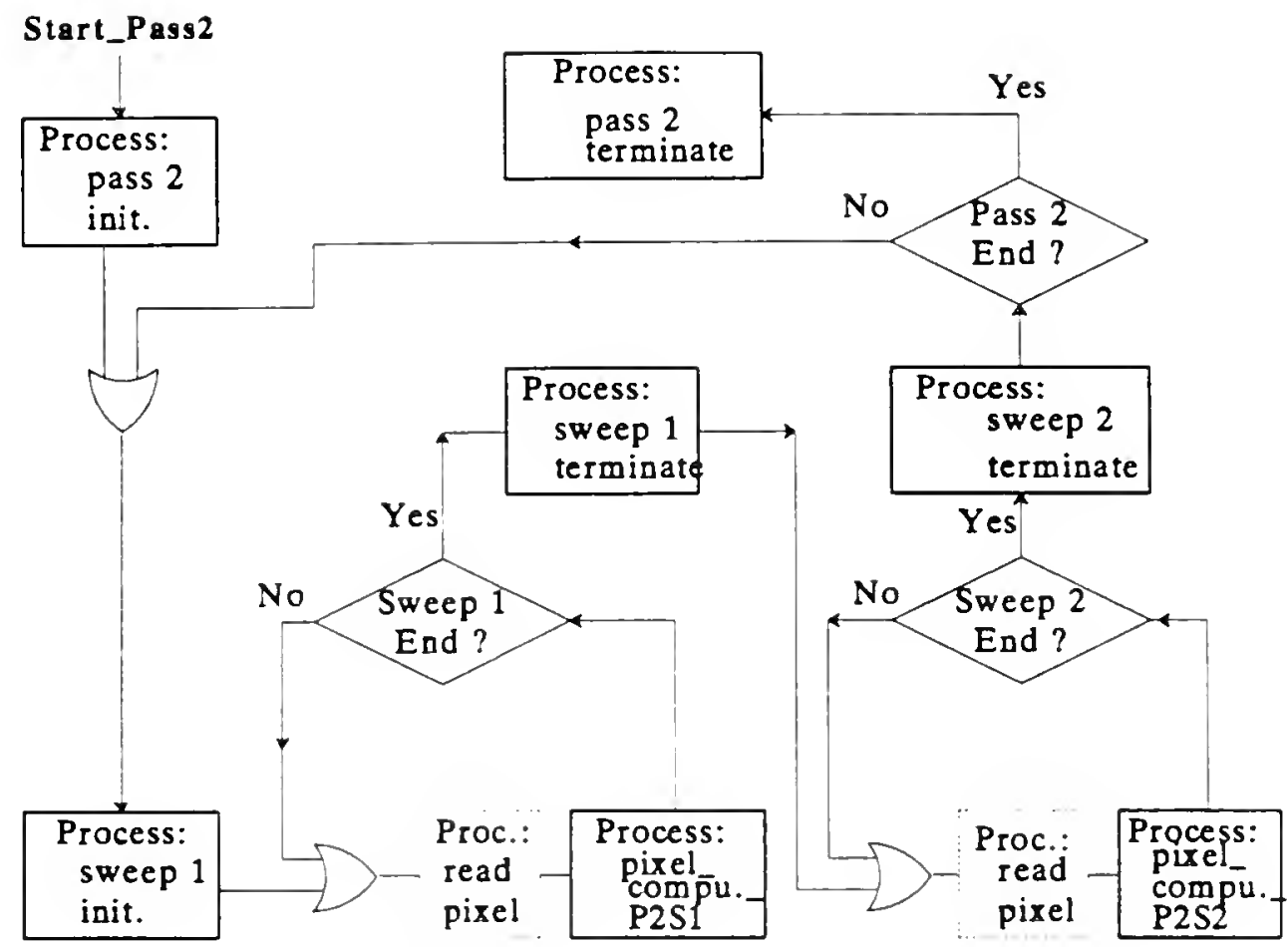

Figure 3.4 The control unit of Pass 2 processor

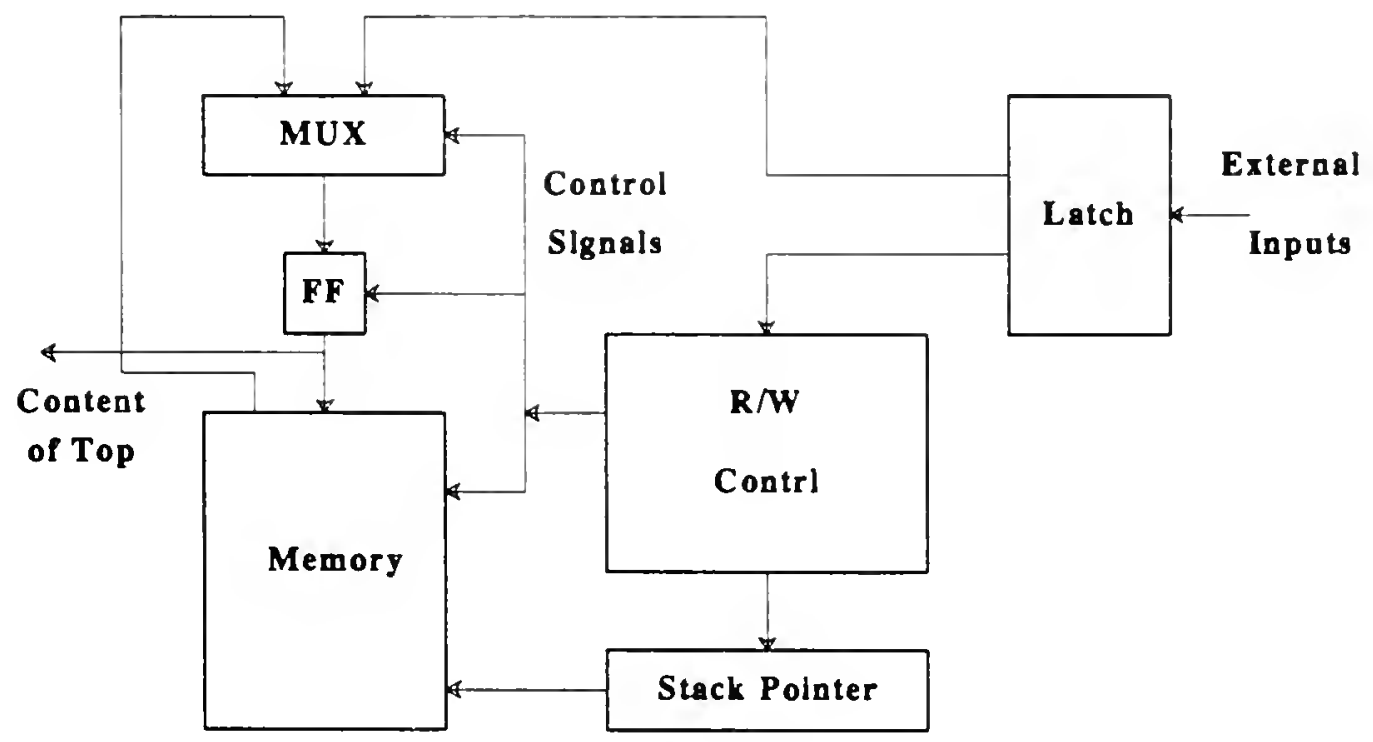

Figure 3.5 The structure of pushdown stack

memory and a counter serving as the stack pointer as depicted in Fig. 3.5. Since the content of the top of a stack is frequently referenced as variables throughout the process, it is stored in a register rather than in memory. A push stack operation is performed in three steps: 
(1) Increment stack pointer by one.

(2) Write the content of the register (the last top entry) into memory.

(3) Gate the external value (the new top entry) into the register.

The pop operation is done in a similar manner. The updating of the top entry of a stack can be most efficiently done by just gating the external input to the register.

Note that since the stack operation needs more than one clock cycle, we have to latch the external input and control signals to the stack during the whole stack operation. Such latching circuits can be avoided if we design a stack in VLSI by just a simple array of bi-directional shift-register, since any operation needs only one cycle in this case.

\subsubsection{Image Buffer}

The image buffer is for storing a $512 \times 5121$-bit binary image whose structure is shown in Fig. 3.6. Because of the relative low speed of memory access, we compact 8 bits into one word. So we need a total of $32 \mathrm{~K}$ bytes memory. While reading pixels, a word is loaded from the memory into an 8-bits shift-register. Then individual bits can be get at the output pin of the shift-register sequentially. The advantage of this is that we have only one memory access for every eight pixels. In addition, the loading of a word from memory to shift-register could be overlapped with pixel computation.

As we mentioned in the algorithm, we need to scan two rows simultaneously.

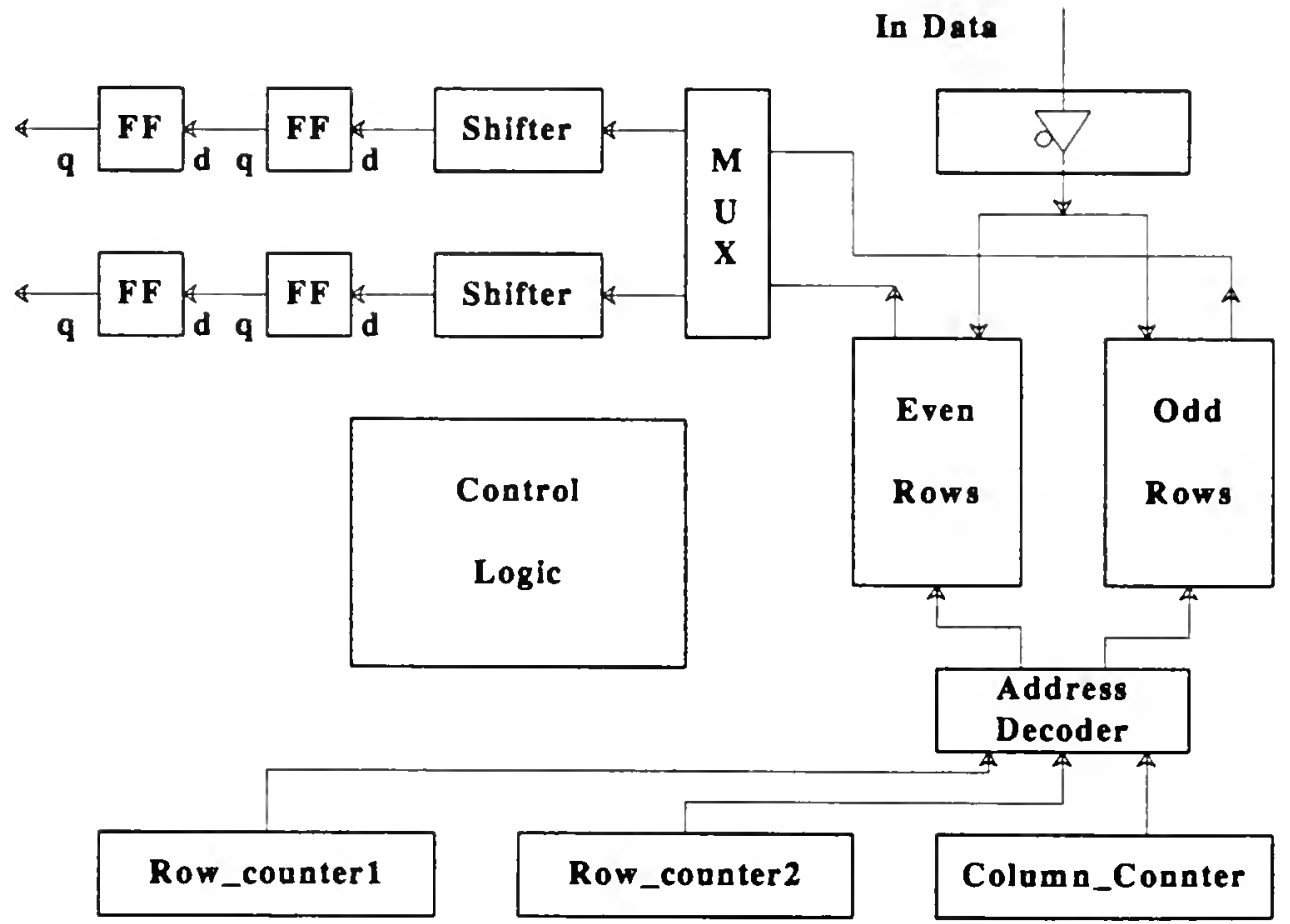

Figure 3.6 The Structure of Image Buffer 
Since the two rows that we want to read at the same time are always consecutive, one is an even numbered row and the other is odd numbered. This suggests a natural division of the image buffer into two ranks, one for even rows and one for odd rows, so we don't have conflicts in reading two consecutive rows at a time. The pixels from the current row and the previous row are obtained at out pins of shiftregisters and latched in D-ff's.

Row_counterl, row_counter2, and column_counter provide the effective addresses for accessing two memory ranks through an address decoder.

\subsubsection{Bracket Table}

The bracket table is shown in Fig. 3.7. The bracket markings are encoded by two bits. These two bits are stored in two ranks of memory, $512 \times 256 \times 1$ bits each, for left-and right-bits respectively. The current bracket markings, read from the table, are latched in two D-ffs.

The row number is provided by row_counterl and row_counter 2 through a multiplexer. The column number is chosen from run_counter 1, run_counter 2 , the top of stack 2 or last popped entry from stack2. An address decoder generates effective addresses for both ranks.

\subsection{Input/Output Interface}
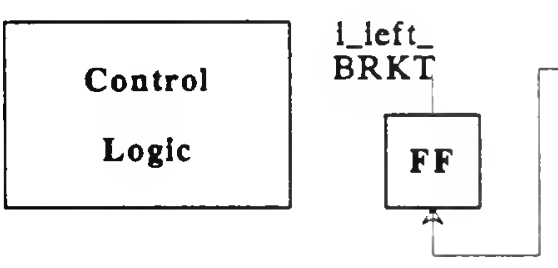

left_BRKT
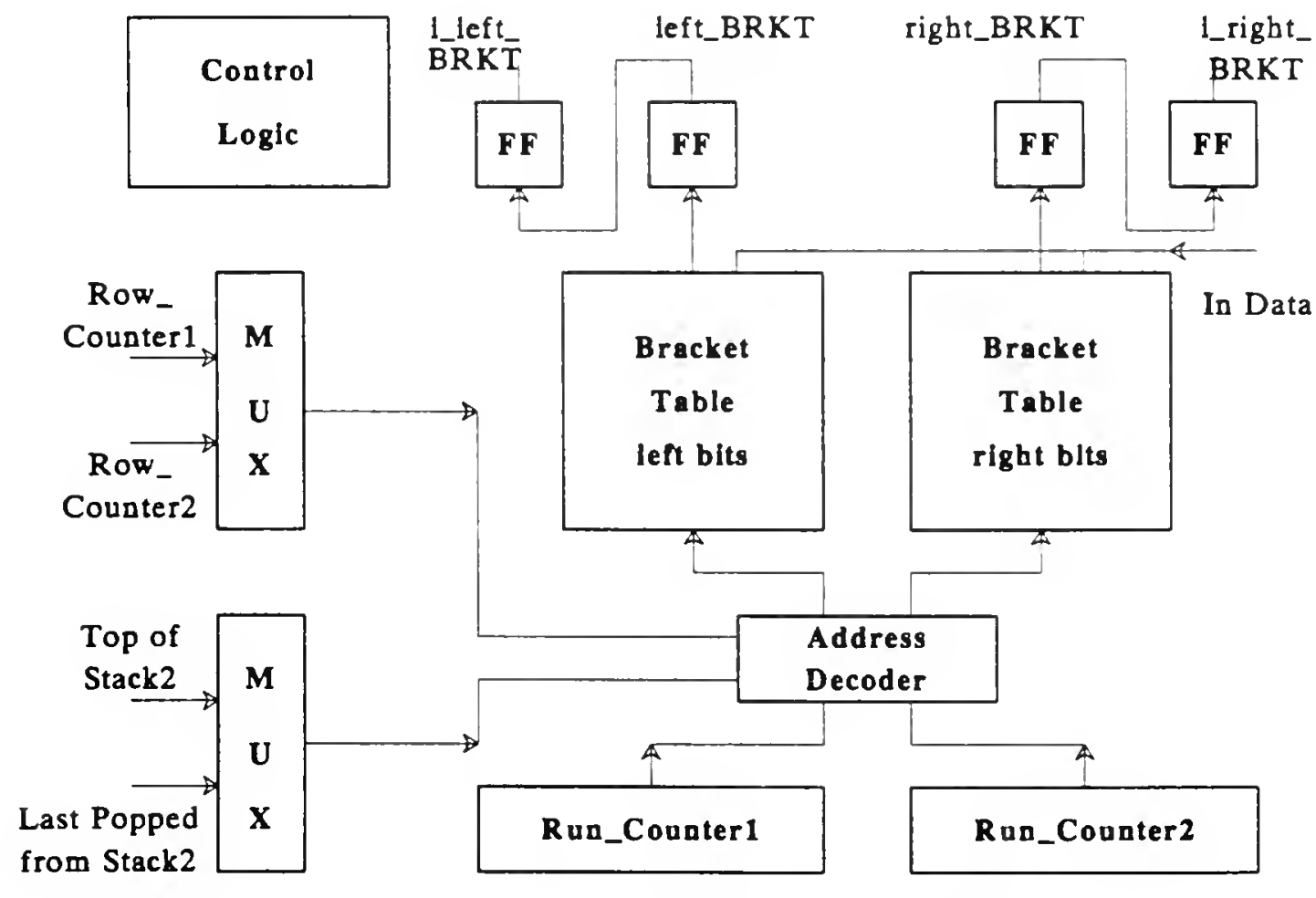

Figure 3.7 The structure of bracket table 
The general purpose handshaking interface we designed is flexible enough to be attached to a DMA controller, a parallel port or a serial port easily with a little additional logic.

The block diagram of the interface is shown in Fig. 3.8. There are two registers: (1) a status register; and (2) a command register. The meaning of each bit in the status register is given in Fig. 3.9(a) and in the command register in Fig. 3.9(b). The data bus has eight bit bi-directional lines. In the write-to-board mode, the data is supplied by an external device; and in read-from-board mode, the data bus is set

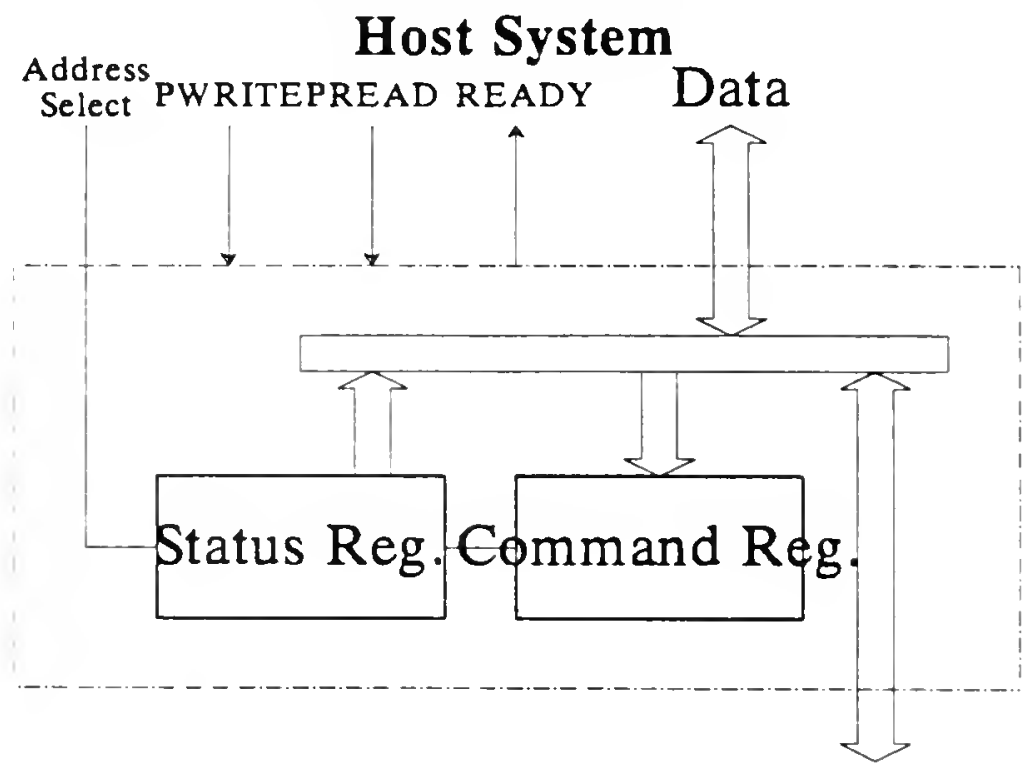

Connected Components Board

Figure 3.8 The block diagram of interface

Status Register
\begin{tabular}{|l|l|l|l|l|l|l|l|}
\hline 7 & 6 & 5 & 4 & 3 & 2 & 1 & 0 \\
\hline
\end{tabular}

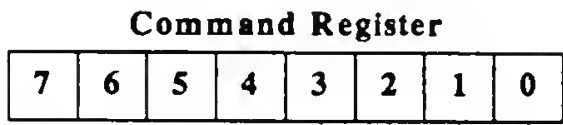

L In Down Load

In Pass 1

In Pass 2

In Pass 2 Sw eep 1

Reset

In pass 2 Sweep 2

Start Pass 1

Start Pass 2

- All bits are high active.

Figure 3.9 Bits assignment in status and command register 
by the board. The data transfer direction is controlled by two input lines, PREAD and PWRITE, which are low active and exclusive. Another output line from the board, called READY, is an acknowledge signal to an external device to indicate that the buffer register of the board is empty in write-to-board mode, and full in read-from-board mode. The signal READY is high active.

The timing of those signals for $\mathrm{I} / \mathrm{O}$ operations is given in [10].

\section{Considerations on VLSI design and Real-time Applications}

Since the connected components algorithm is such a fundamental procedure in image analysis and has more and more applications in robot vision, it is very attractive to implement this design on a VLSI chip, so it can be conveniently added to an image processing computer or general purpose system. Although we can simply map the whole circuit onto a chip and replace each logical gate by a corresponding cell of VLSI technique, it is not the best way to do it. The disadvantage of this approach is that the irregular structure of original design makes the physical layout in VLSI difficult. Many of the components can be implemented instead in a more efficient and much simpler style of VLSI.

For instance, a pushdown stack can be implemented by an array of bi-directional shift registers. It has several advantages: (a) it has a very regular pattern, so it is extremely simple to change the dimension of a stack; (b) a stack pointer is no longer necessary; (c) each of the three kinds of operations on a stack can be performed in just one cycle; (d) because of (c), the latching circuit for external inputs and control signal to the stack can be eliminated.

A control unit in VLSI is usually implemented in a more regular structure rather than a random logic design. The typical structure of a finite state machine in VLSI

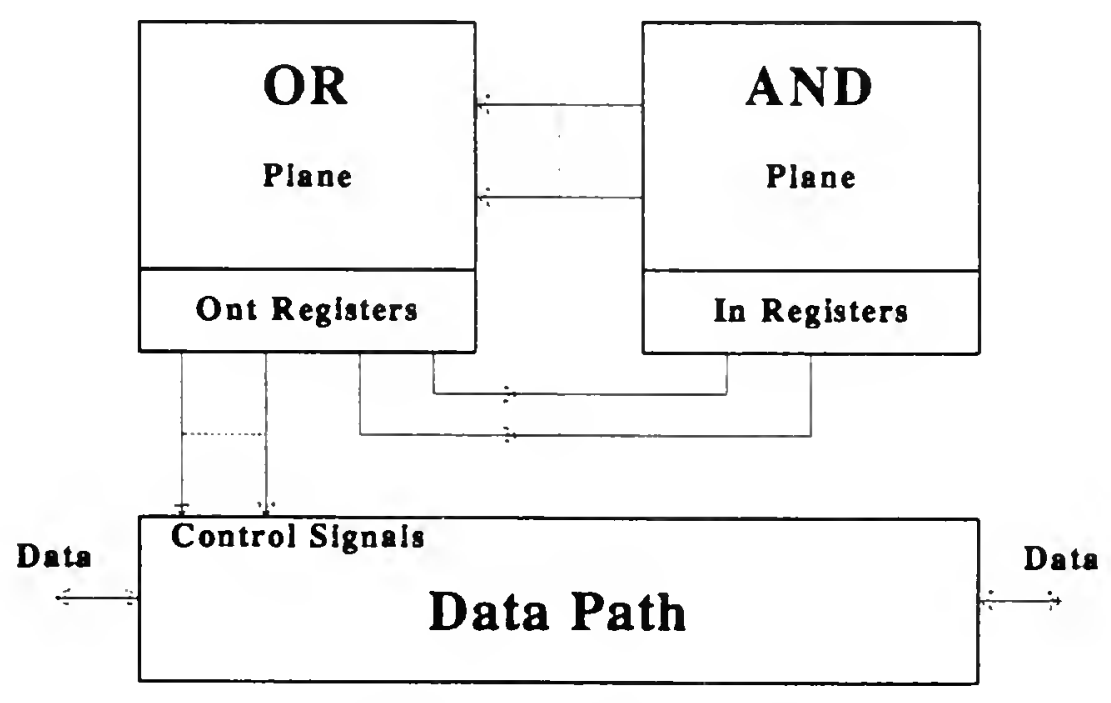

Figure 4.1 Finite state machine 
is shown in Fig. 4.1. The combinational logic network implements logical functions in the form of

$$
Z=\sum_{j=1}^{n} a_{j_{1}} \cdots a_{j_{m}}
$$

It is not very difficult to write down the input to a register in our design as the function of other variables in the above form. So the full VLSI implementation is obviously possible.

More parallelism can be obtained at both the global and local level. For example, in the above design as Pass 1 processor is running, Pass 2 processor is idle. Globally, it is valuable to pipeline the two processors when we need to compute the input images continuously. A possible configuration is shown in Fig. 4.2. Instead of putting whole system on one chip, it can be naturally divided in to four modules, namely the pass 1 processor, the pass 2 processor, the image buffer, and the bracket table. In Fig. 4.2 we have two image buffers and two bracket tables. When the pass 1 processor is working on set 1 , the pass 2 processor works on set 2 . At the end of a pass, the two processors can exchange image buffers and bracket tables. So when the pass 1 processor finishes one pass on image 1 , the result is stored in bracket table 1. Then these data are passed to the pass 2 processor and at the same time the pass 1 processor starts working on the next incoming image with the other image buffer and bracket table. Data switching can be done trivially with multiplexers.

If the order of output sequence of a symbolic image is allowed to be reversed, we can reverse the sweeping directions of both pass 1 and pass 2 . In such a case, the pass 1 processor doesn't have to wait until the entire image has been loaded into the image buffer. In other words, it can run during the image loading process. This, however, introduces the possibility of simultaneous accesses to the image buffer by the image loading process and the pass 1 computation process. So some additional mechanism, such as a semaphore, is needed to coordinate the processes.

Locally, some operations in each of the processes can be obviously performed during a single cycle without conflicts in the data path. Such parallelism can be

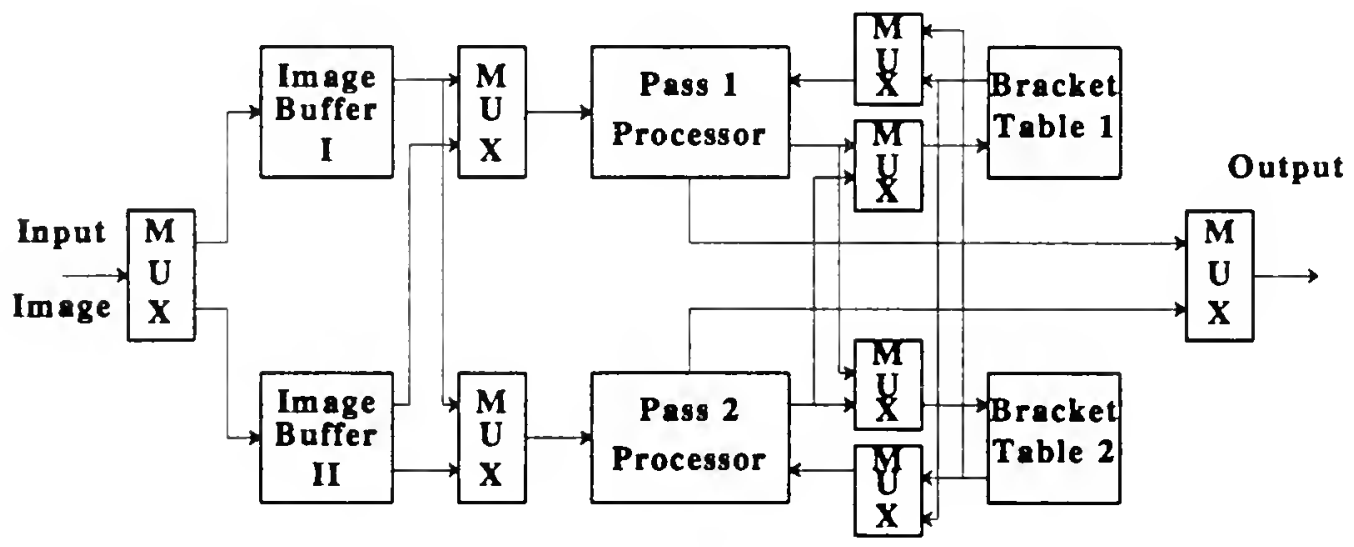

Figure 4.2 The configuration of a pipeline processor 
easily achieved by broadcasting a control signal to more than one proper data components. This kind of parallelism has been realized in the many processes of our design.

As the speed of computation increases, data transfer will cause a bottleneck. It seems that regular DMA transfer in micro or mini systems would not be able to match the the computation speed. An ultra fast secondary bus directly connecting it to other system modulars is more preferable.

\section{Acknowledgements}

I wish to thank Prof. Jack T. Schwartz and Prof. Alan Siegel for their explanations of the algorithm to me. I had very valuable discussions with $\mathrm{A}$ lan $\mathrm{Kalvin}$ and Eric Freudenthal in considering the algorithm details. I also like to thank Prof. H. Stone for reviewing my design and giving me some valuable suggestions. Special thanks to F. Hansen, S. Tavana, D. Clark, and J. Fehlinger who gave me a lot of technical assistance in building the connected components board.

\section{References}

[1] Ballard, D.H., Brown, J.E., Computer Vision, Prentice-Hall, Inc., NJ, 1982

[2] Dinstein, I., Yen, D., Flickner, M., Handling Memory Overflow in Connected Component Labeling Applications, IEEE Trans. on PAMI, Vol. PAMI-7, No. 1, Jan. 1985

[3] Lumia, R., Shapiro, L., Zuniga, O., A New Connected Components Algorithm for Virtual Memory Computers, Computer Vision, Graphics, and Image Processing 22, 287-300, 1983

[4] Rosenfeld, A., Adjacency in Digital Pictures, Information and Control, Vol. 26, No. 1, Sept. 1974

[5] Rosenfeld, A., Kak, A.C., Digital Picture Processing, Vol. I, 2, Academic Press, 1982

[6] Rosenfeld, A., Picture Processing: 1975, Computer Graphics and Image Processing, 5, 215-237, 1976

[7] Rosenfeld, A., Pfaltz, J.L., Sequential Operations in Digital Processing, JACM, 13, 471-494, 1966

[8] Schwartz, J.T., Sharir, M., Siegel, A., An Efficient Algorithm for Finding Connected Components in a Binary Image, Technical Report No. 154, Courant Institute, NYU, 1985

[9] Shiloach, Y., Vishkin, U., An O(log n) Parallel Connectivity Algorithm, Journal of Algorithms 3, 57-67, 1982

[10] Yang, X.D., Connected Components Board Design's Documentation, In preparation, Robotics Lab., Courant Institute, NYU, 1987

[11] Mano, M.M., Computer System Architecture, 2nd ed., Prentice.Hall, Inc., NJ, 1982

[12] Mead, C., Conway, L., Introduction to VLSI Systems, Addison-Wesley, 1980

[13] Weste, N.H.E., Eshraghian, K., Principles of CMOS VLSI Design, Addison-Wealey, Reading, MA, 1985 
Appendix.A

\section{Connected Components Algorithm}

\section{Implementated in C Programming Language}

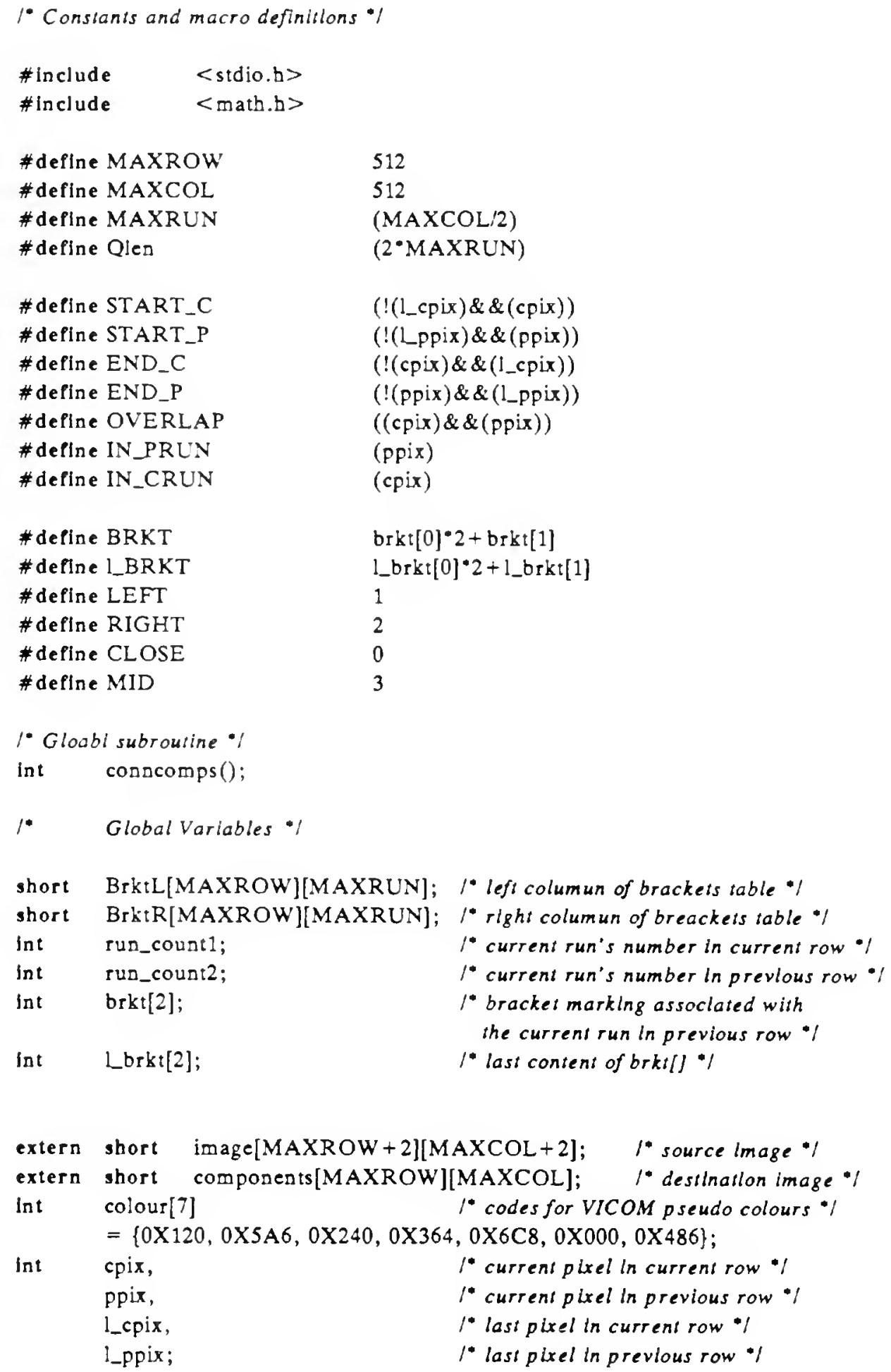


Int queue_count1,queue_count2;

Int component_No;
1 components number queue poincers $\%$

1* next new identify number */

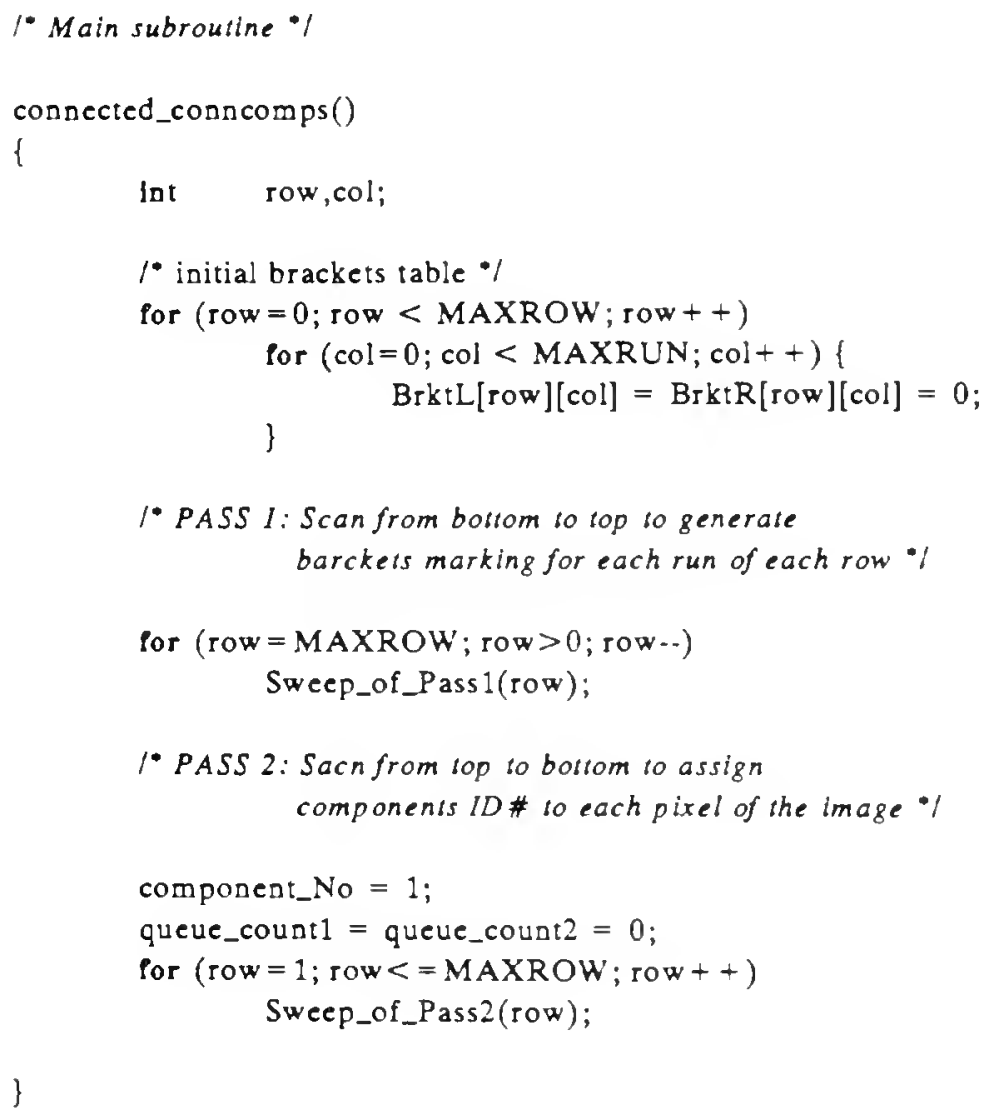




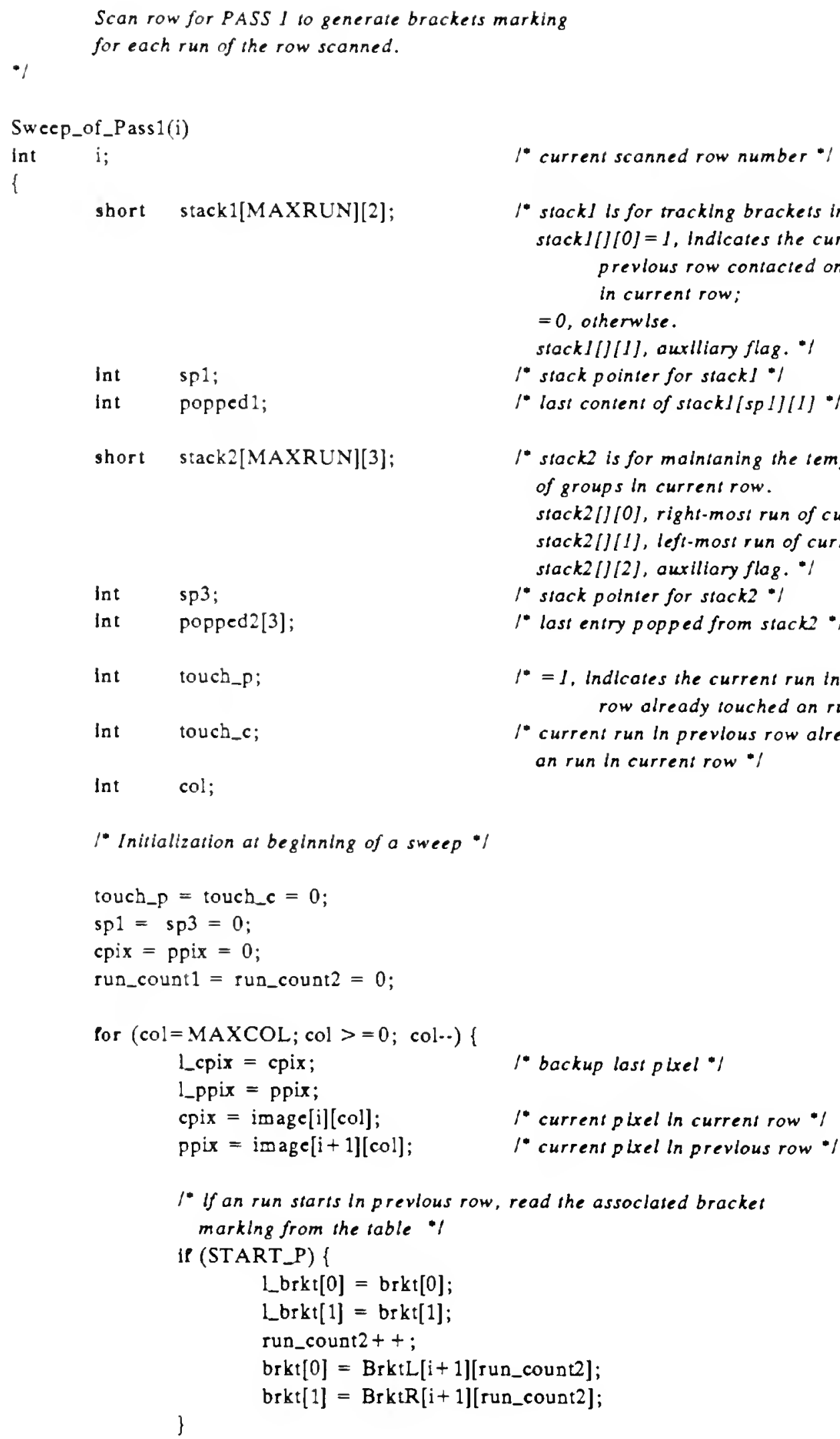




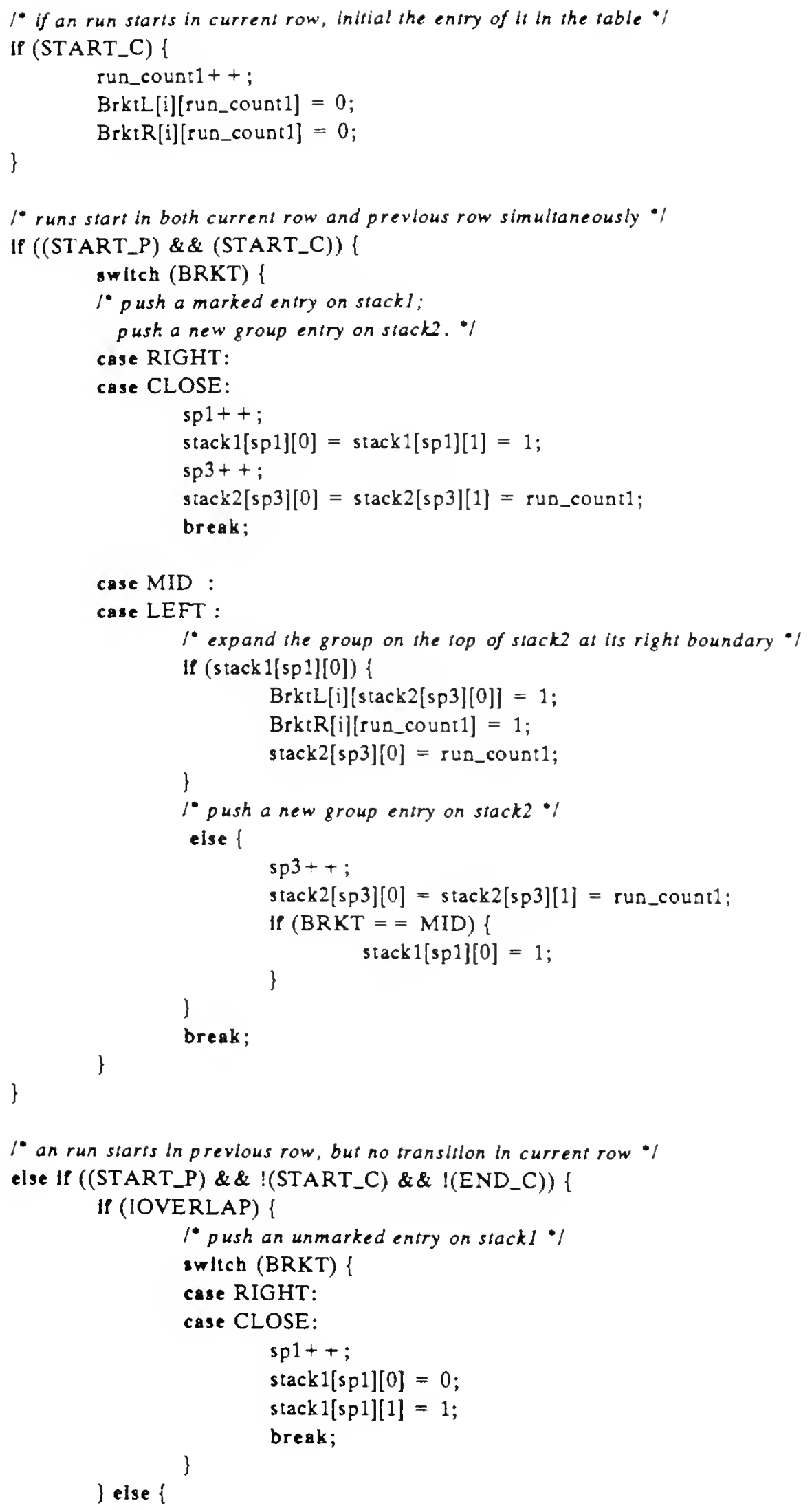




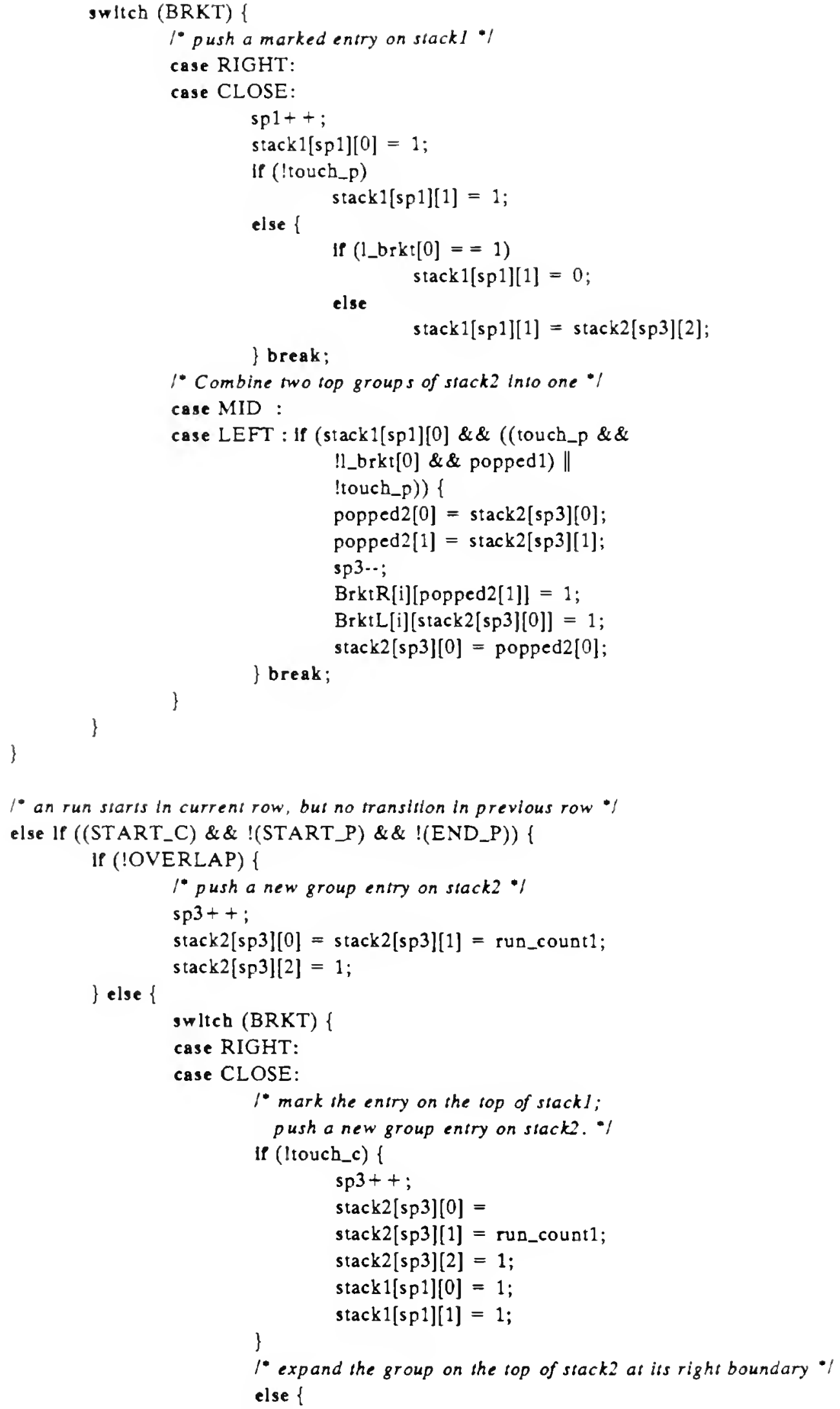




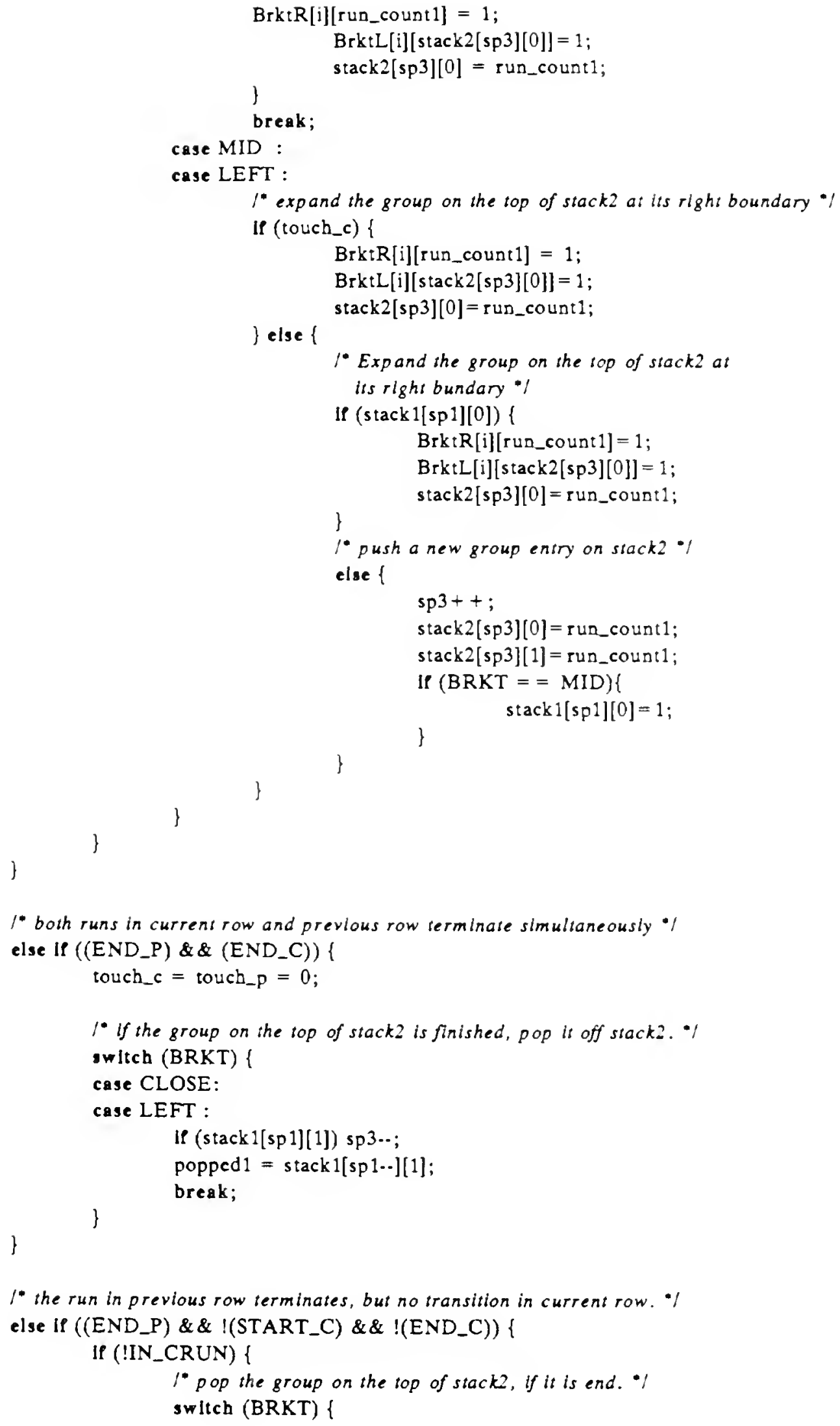




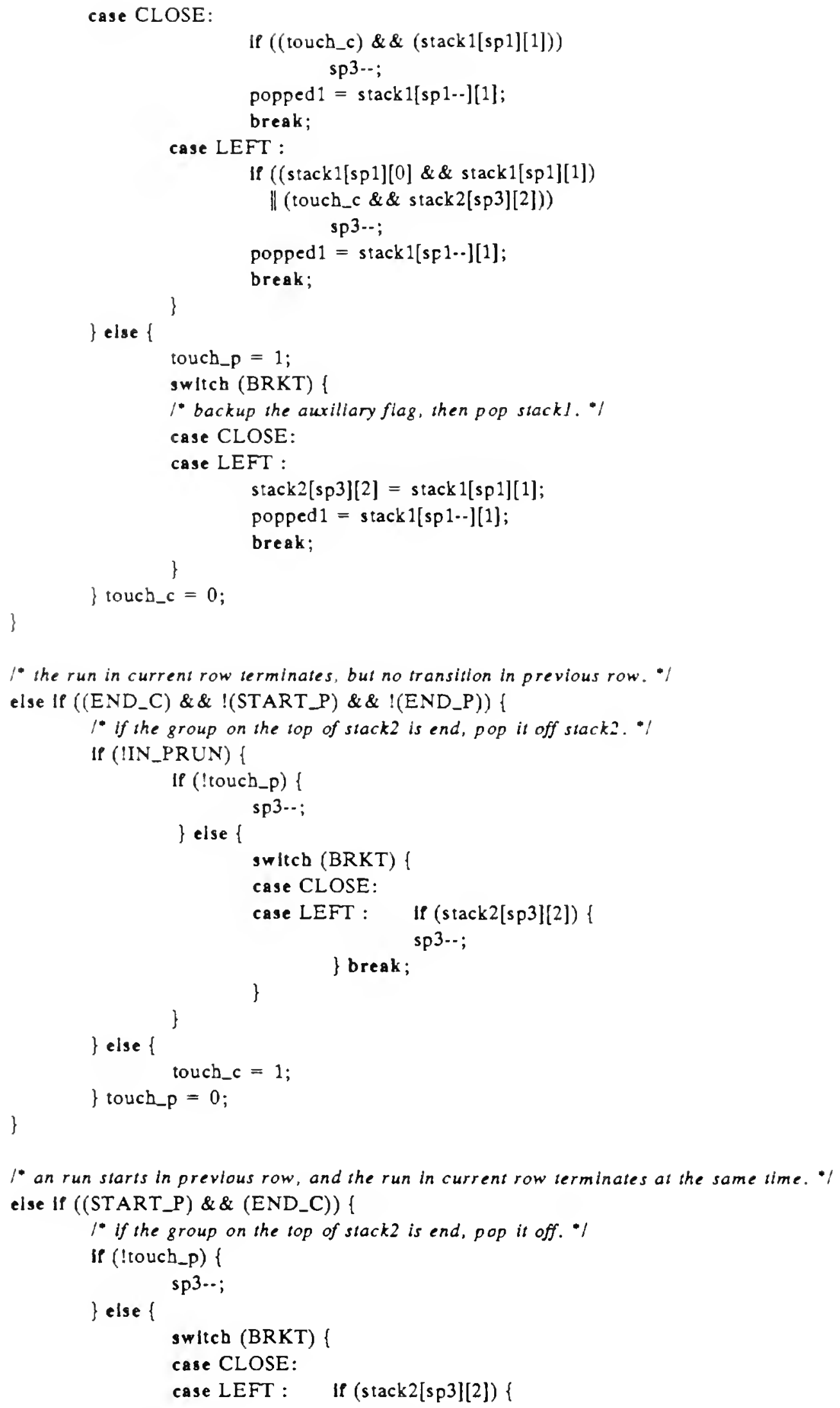




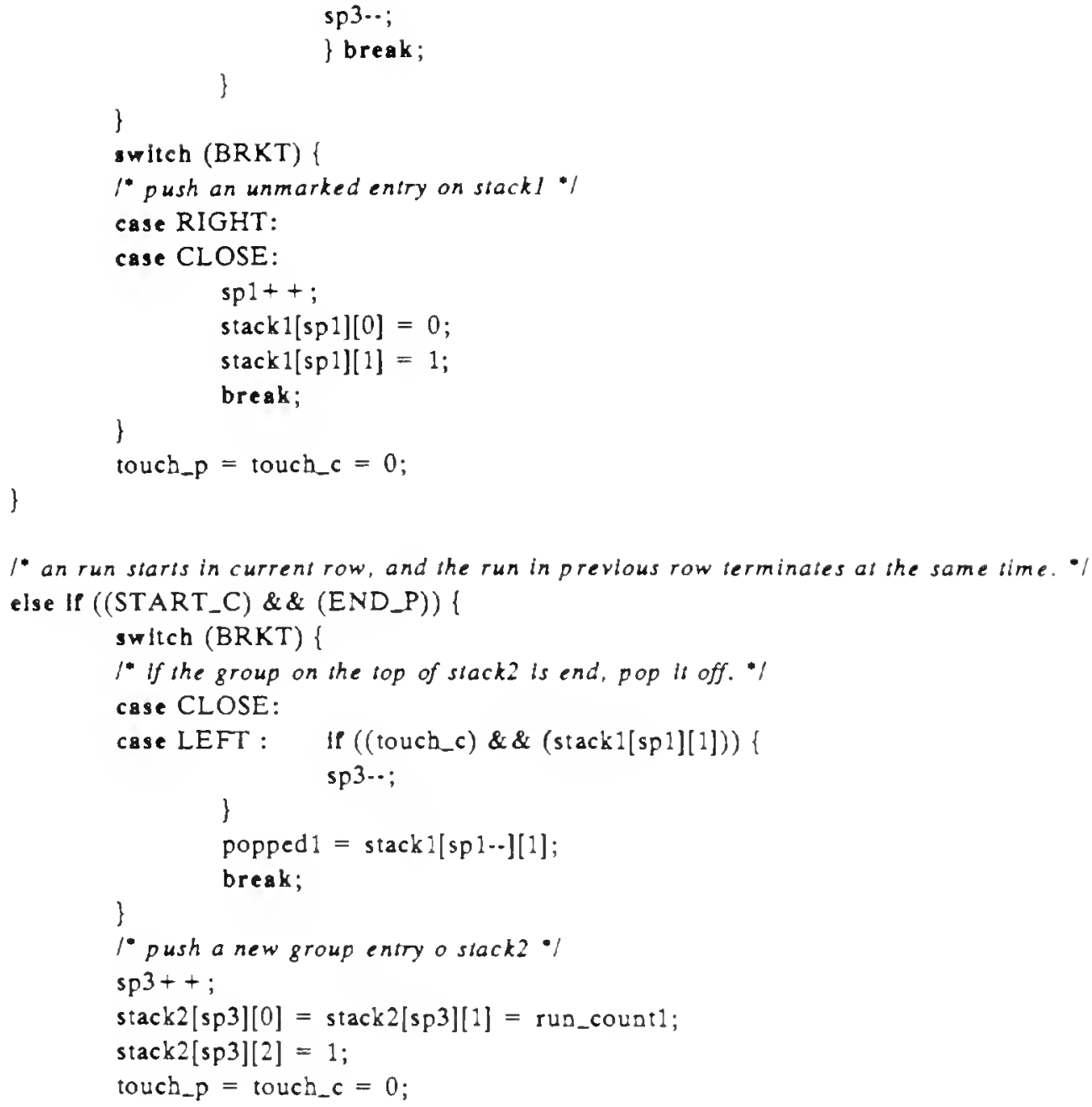




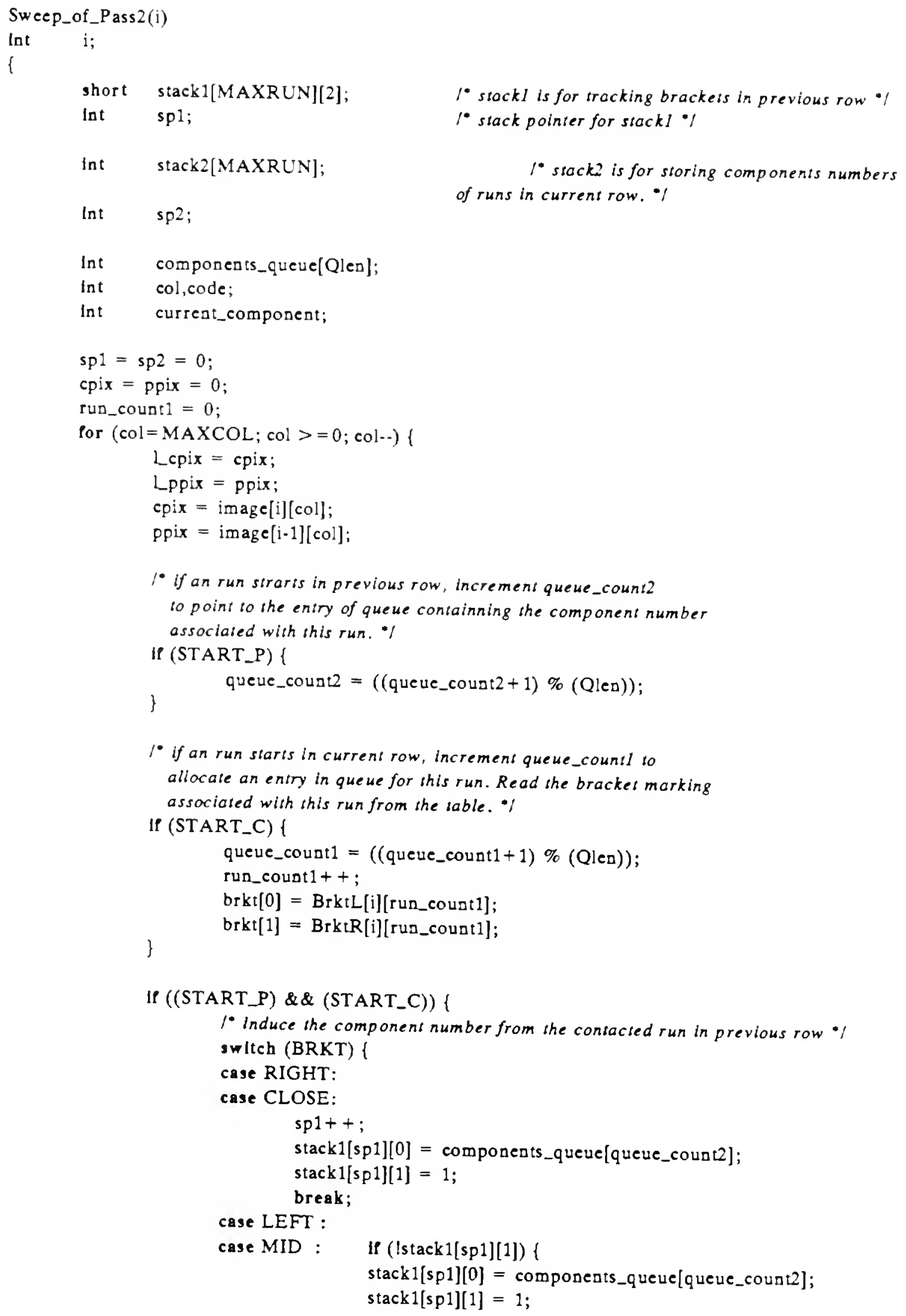




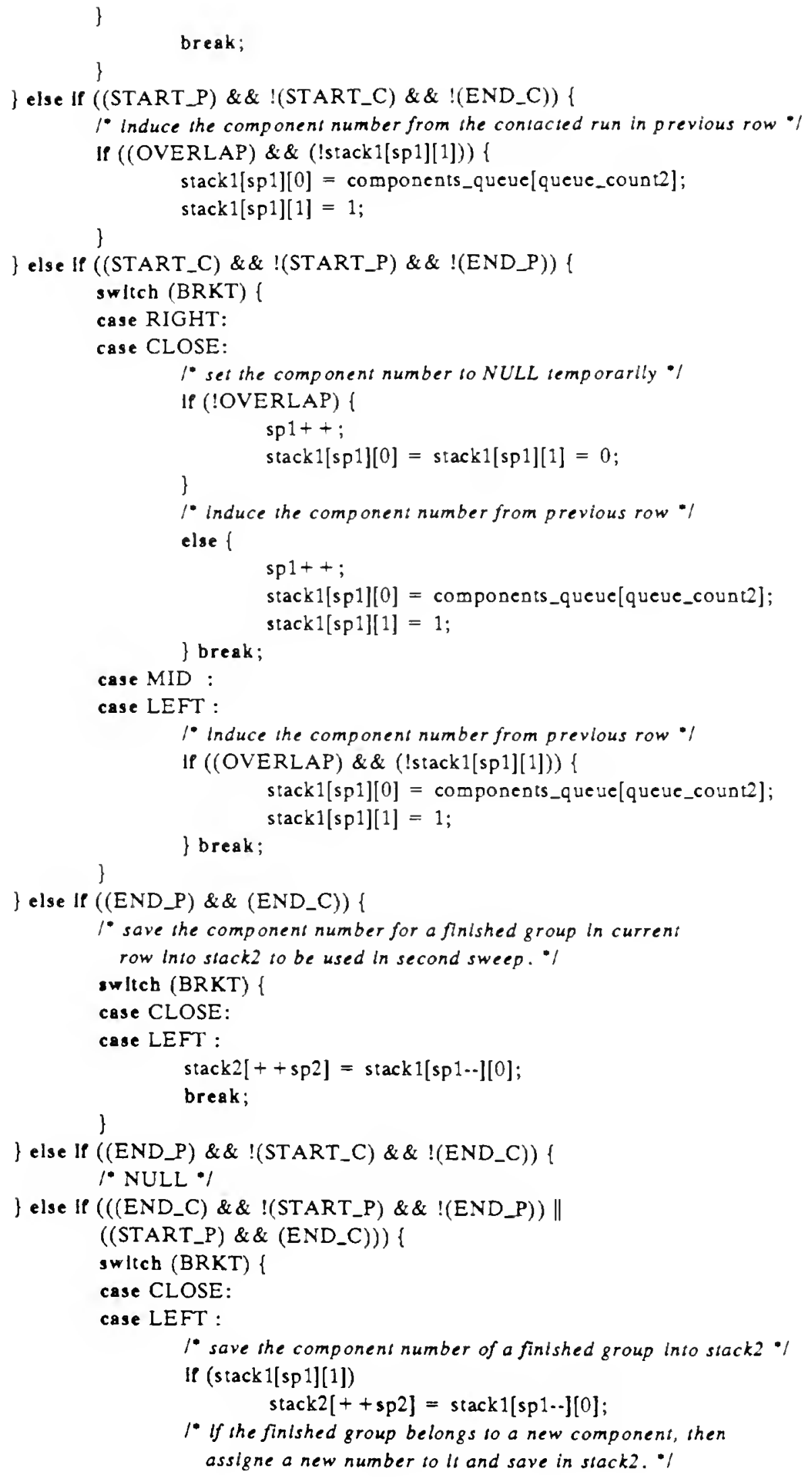




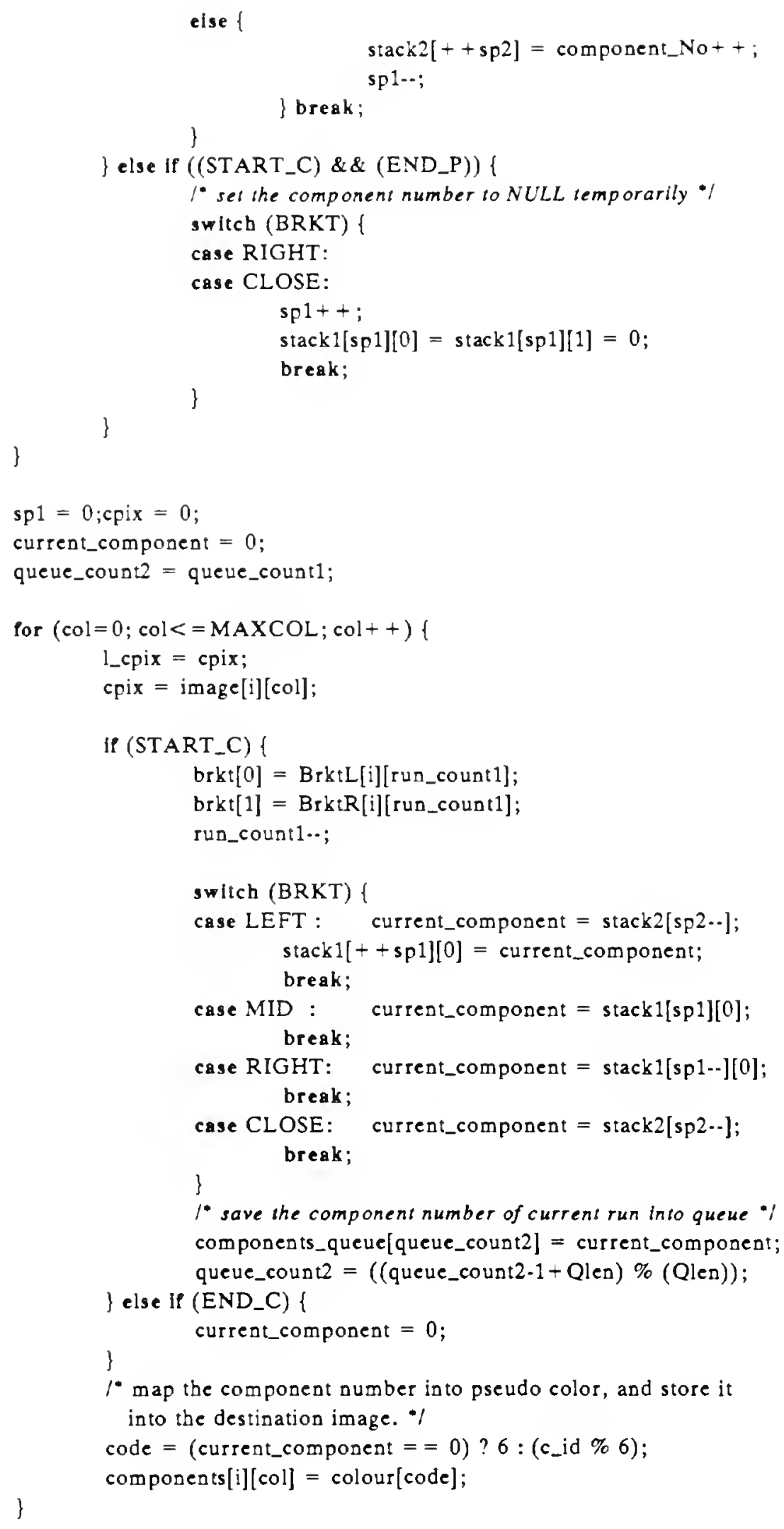


Appendix.B

Process Description of The Algorithm in

A Microparallel Hardware Design Language

\section{B.1 Definitions of Variables and Flags}

image_buffer(0..511,0..511);

Left_bracket_table $(0 . .511,0 . .255)$;

Right_bracket_table $(0 . .511,0 . .255)$;

Row_Count1;

Row_Count2;

Column_Count;

Bracket_Count1;

Bracket_Count2;

P1_Stack1 $(256,2)$;

P1_Stack2 $(256,3)$;

P2_Stack1 $(256,2)$;

P2_Stack2 $(256,1)$;

S1_popped(2);

S2_popped(3);

Queue(0..512);

Queue_Count 1;

Queue_Count2;

CPIX;

1_CPIX;

PPIX;

L_PPIX;

brkt(2);

L_brkt(2);

touch_c;

touch_p;

In_Down_load;

In_Pass 1;

In_Pass2;

Start_pass 1;

Siart_pass2;

Sweep2_of_Pass2_enable;
1* The image storage buffer is divided into two ranks, one for even rows and one for odd $\%$

1" Table of left bracket bits "/

1" Table of right bracker bits"/

1" 9 bits: binary up-down counter "/

1" 9 bits: Binary up-down counter */

1" 9 bits: binary up-down counter "/

1" 8 bits: up-down counter "

1" 8 bits: up-down counter"/

1* Pushdown stack holds two 1-bit fields; dep th $256 \%$

1* Pushdown stack holds three fields; depth $256^{\circ} /$

1"Pushdown stack holds two fields; depth $256^{\circ} /$

1* Pushdown stack holds one field; depth 256 \%

1* Lasi value popped from P1_Stackl */

/"Last value time popped from P1_Stack2 "/

/* Circular queue */

1* 9 biss: counter pointing to front of queue */

1" 9 bits: counter pointing to end of queue $\%$

1* I bis: current pixel of current row $*$

1" I bit: previous pixel of current row $" /$

1* 1 bit: current pixel of previous row $\%$

/* 1 bit: previous pixel of previous row */

1* 2 bits: current brackets $\%$

1" 2 bits: previous brackets "।

1* 1 bit: flag used in PASS 1*/

1* I bit: flag used in PASS $1 \%$

1* flag: indicates down loading in progress "/

1* flag: indicates Passl in progress "/

1* flag: indicales Pass 2 in progress */

1* flag: enables process Initialization_of_Sweep_of_PassI *

1* flag: enables process P2S1_Row_Initialization $\%$

/* flag: enables process P2S2_Row_Initialization */ 
Start_Row_Sweep_of_Pass1;

Start_Row_Sweep1_of_Pass2;

Start_Row_Sweep2_of_Pass2;

Next_Row_P1;

Next_Row_P2;

Next_Pixel_1;

Next_Pixel_2;

Next_Pixel_3;

P1_pixel_op_enable;

P2S1_pixel_op_enable;

P2S2_pixel_op_enable;

component_number;

current_component;
1* flag: enables process Read_Pixel */

/* flag: enables process Read_Pixel */

1* flag: enables process Read_Pixel */

1* flag: enables process Initialization_of_Sweep_of Pixel $\%$

1* flag: enables process P2SI_Row_Initialization */

I* flag: enables process Read_Pixel */

I* flag: enables process Read_Pixel */

1*flag: enables process Read_Pixel */

/"flag: enables process Pixel_Computation_of_Pass1"/

1* flag: enables process Pixel_Computation_of Sweep_of_Pass?

/* flag: enables process Pixel_Computation_of_Sweep_of_Pass?

1" Next new component number "/

/* component number of current scanned pixel */

\section{B.2 Description of Primitive Operations}

1. Assigning a value to a simple variable or indexed variable has obvious meaning.

2. Four stack operations are used:

$$
\begin{aligned}
& \text { push(stack_name, vector); } \\
& \text { pop(stack_name); } \\
& \text { /* push a vector onto multi-field } \\
& \text { stack" } \\
& \text { top }(\text { stack_name })=\text { vector; } \\
& \text { I" pop the top of a stack "/ } \\
& \text { top }(\text { stack_name })[i]=\text { variable; } \\
& \text { 1* modify the top of a stack"/ } \\
& \text { 1* modify a specific field of } \\
& \text { a stack"। }
\end{aligned}
$$

3. Queve operations are treated as indexed accesses to an underlying array.

\section{B.3 Processes}

/" Initialize row counters for Pass I then start row sweeping process "/

Process PASS1_Initialization

If (Pass1 \& ! In_Down_Load \& !In_Pass1 \& !In_Pass2) then

In_Pass 1 = true;

Row_Count1 = 511;

Row_Count2 = 0;

Start_Pass1 = true;

else

Endif;

$$
\text { Start_pass1 = false; }
$$


" Terminate Pass 1 afier all rows have been scanned *

Process PASS1_Termination

if (In_Pass1 \& Next_Row_P1 \& Row_Count1= =511) theo

Endif; In_Pass 1 = false;

1* Initiallze column counser variables and flags at entrance of a row sweep In Passl then enable reading pixel process *

Process Initialization_of_Sweep_of_Pass 1

If (Start_Pass1 | Next_Row_P1 \& Row_Count1 != 511)) then

Nexi_Row_P1 = false;

Column_Count $=511$;

reset( $\mathrm{P} 1 \_$stack 1$)$;

reset(P1_stack2);

CPIX $=0$;

$\mathrm{PPIX}=0$;

touch_p $=0$;

touch-c $=0$;

Bracket_Count1 $=0$;

Bracket_Count2 =0;

else

Start_Row_Sweep_Pass 1 = true;

Endif;

Start_row_Sweep_Pass1 = false;

1* At the end of each sweep, update row counters, and start sweep on next row "

Process End_of_Sweep_of_Pass1

If (Next_Pixel_1 \& Column_Count $=511$ ) then

Row_Count 1 ..;

Row_Count2 ...;

Next_Row_P1 = true;

Endif;

1* Load next pixels from current row and prevlous row simulianeously into regisiers CPIX $P P I X$ respectively, and old conients of $C P I X$ and $P P I X$ was backed into l_CPX and l $P P I X$ respectively. If a run staried in a prevlous row, the corresponding bracket marking is loaded from bracket table. $\%$

Process Read_Pixel

1* In sweep 1 of pass 1: 
Recd plxels from two rows simultaneously from right to left.

$\rightarrow$

If (Start_Row_Sweep_of_Pass1|Next_Pixel_1 \& Column_Count != 511) then

Next_Pixel_1 = flase;

1_CPIX = CPIX;

1_PPIX = PPIX;

CPIX = image_buffer(Row_Count1,Column_Count);

PPIX = image_buffer(Row_Count2,Column_Count);

Column_Count ...;

If $(P P I X=1 \& L P P I X=0)$ then

Lbrkt[left] = brkt[left];

Lbrkt[right] = brkt[right];

Bracket_Count $2++$;

brkt[left] = Left_bracket_table(Row_Count2,Bracket_Count2);

Endle;

brkt[rigbt] = Rigbt_bracket_table(Row_Count2,Bracket_Count2);

If $(\mathrm{CPIX}=1$ \& $L \mathrm{CPIX}=0)$ then

Bracket_Count1 + + ;

Left_bracket_table(Row_Count1,Bracket_Count1) =0;

Endif;

Right_bracket_table(Row_Count1,Bracket_Count1) =0;

P1_pixel_op_enable = true;

1* In sweep 1 of pass 2:

Read pixels from two rows simultaneously from right to left.

!

else If (Start_Row_Sweep1_of_Pass2 |Next_Pixel_2 \& Column_Count != 511) then

Next_Pixel_2 = false;

1_CPIX = CPIX;

LPPIX = PPIX;

CPIX = image_buffer(Row_Count1,Column_Count);

PPIX = image_buffer(Row_Count2,Column_Count);

Column_Count ...;

If (PPIX = 1 \& 1_PPIX =0) then

Queue_Count2 + + ;

Endif;

If $(\mathrm{CPIX}=1 \&$ LCPIX $=0)$ then

Queue_Count1 + + ;

Bracket_Count $2++$;

brkt[left] = Left_bracket_table(Row_Count1,Bracket_Count2);

Endif;

brkt[right] = Right_bracket_table(Row_Count1,Bracket_Count2);

P2S1_pixel_op_enable = true;

1* In sweep 2 of pass2:

Read plxels from two rows simultaneously from left to rlght.

- 


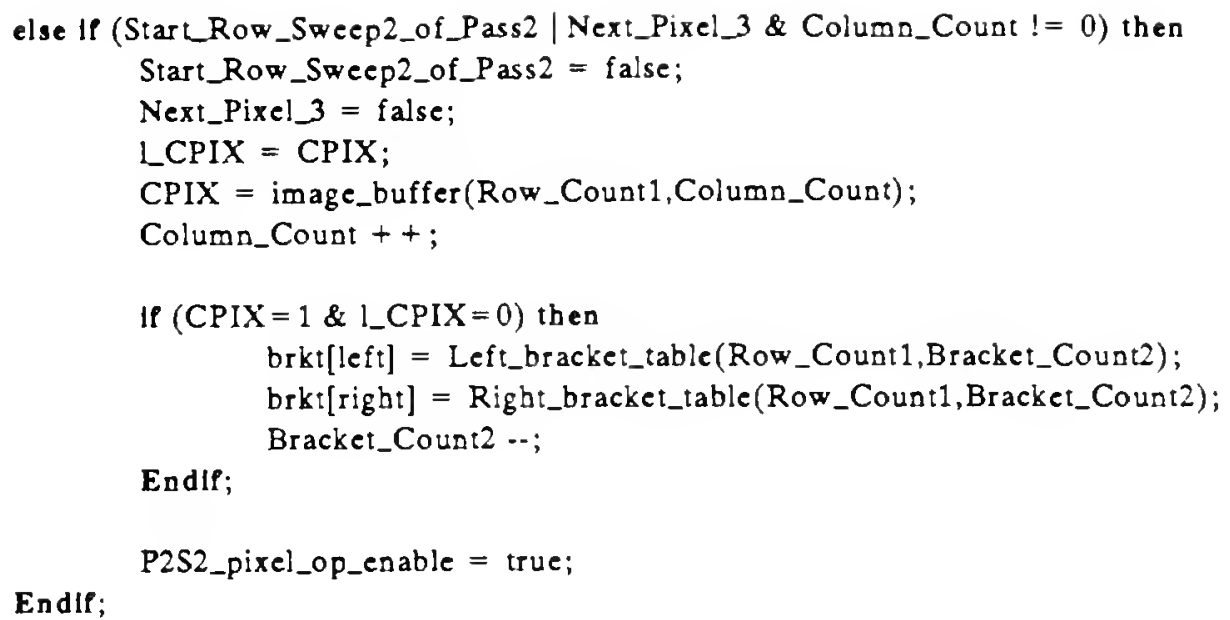

1* this ts the main computation process of Passl. In different cases of combinational conditions approprlate manlpulation are carrled out on two stacks and bracket table. "/

Process Pixel_Computation_of_Pass 1

If (P1_pixel_op_enable) then

P1_pixel_op_enable $=$ false;

case of

1* Start a run on both current and prevlous rows */

$($ PPIX $=1 \&$ LPPIX $=0$ \& CPIX = 1 \& \& !_CPIX =0):

case of

(brkt[right] $=0)$ :

push(P1_Stack1,(1,1));

push(P1_Stack2,(bracket_Count1,bracket_Count1));

(brkt[right] $=1$ ):

If (top(P1_Stack1)[0]) then

Left_bracket_table(Row_Count1,top(P1_Stack2)[0]) =1;

Right_bracket_table(Row_Count1,Bracket_Count1) =1;

else

push(P1_Stack2,Bracket_Count1,Bracket_Count1);

If (brkt[left] = 1) then

Endle:

$\operatorname{top}(\mathrm{P} 1$ Stack 1$)[0]=1$;

Endle;

Endcase;

1* Only start a new run in prevlous row. There ls no

translilon on curtent row. *

$($ PPIX $=1$ \& 1_PPIX $=0$ \& ! (CPIX xor 1_CPIX)):

If !(CPIX \& PPIX) then

case of 


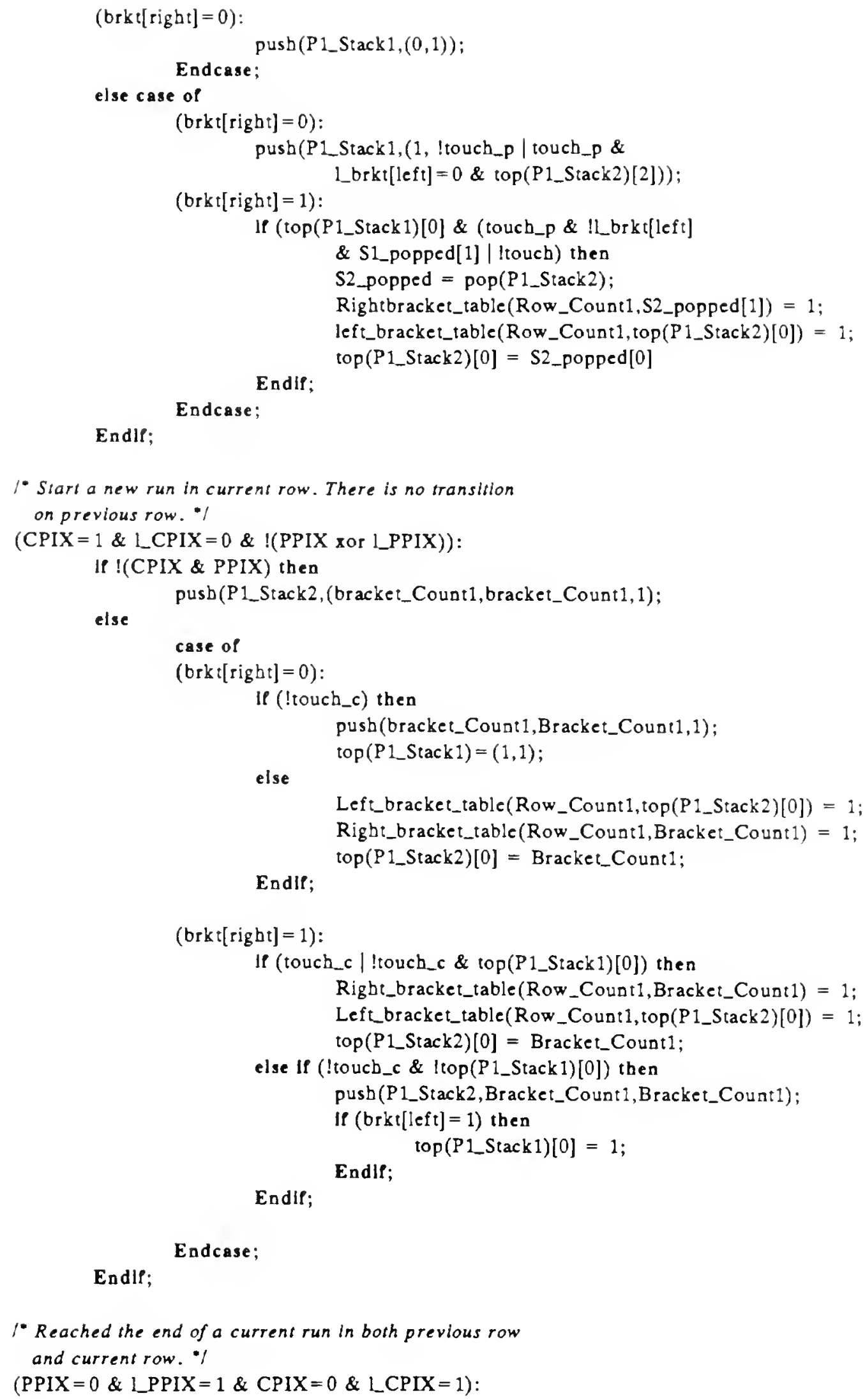




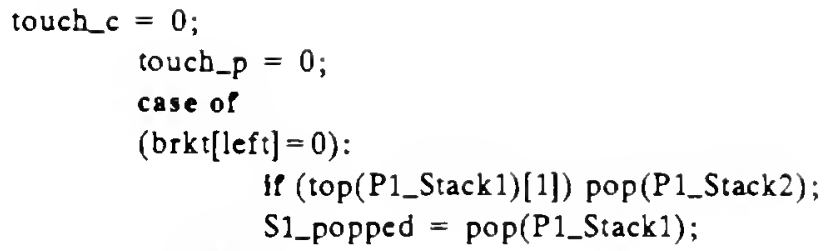

1* Reached the end of a run in previous row. But there is no transition on current row. "

$($ PPIX $=0$ \& L_PPIX $=1 \& !($ CPIX xor LCPIX)):

If (!CPIX) then

case of

(brkt $[$ left $]=0$ \& brkt $[$ right $]=0)$ :

If (touch_c \& top(P1_Stack1)[1]) then

Endip; pop(P1_Stack2);

S1_popped $=$ pop $($ P1_Stack1)

(brkt[left $]=0$ \& brkt[rigbt $]=1)$ :

If (top (P1_Stack1)[0] \& top(P1_Stack1)[1] touch_c \& top(P1_Stack2)[2]) then pop (P1_Stack2);

Endif;

S1_popped $=$ pop $($ P1_Stack1)

\section{Endcase:}

eise

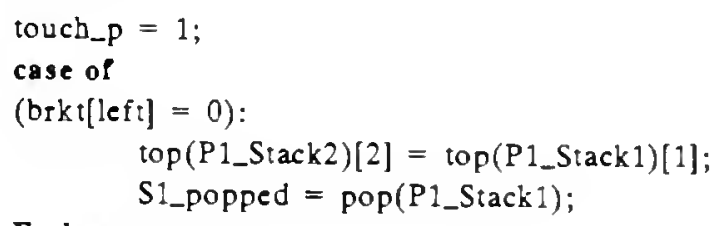

1 Reached the end of a run in current row. But there is

no transition on previous row. $\%$

$($ CPIX $=0$ \& LCPIX $=1$ \& ! (PPIX xor LPPIX)):

If (IPPIX) then

If (ltouch_p $\mid$ touch_p \& brkt[left] $=0$ \& top(P1_Stack2)[2]) then

Endif; pop(P1_Stack2);

else

toucb_c $=1$

Endif;

touch_p $=0$;

\footnotetext{
1* Start a new run in previous row and reach the end of the current run in current row simultaneously. */

(PPIX $=1$ \& LPPIX $=0$ \& CPIX $=0$ \& I_CPIX $=1)$ :

If (!touch_p $\mid$ touch_p \& brkt[left] $=0 \&$ top $\left.\left(P 1 \_S t a c k 2\right)[2]\right)$ then
} 


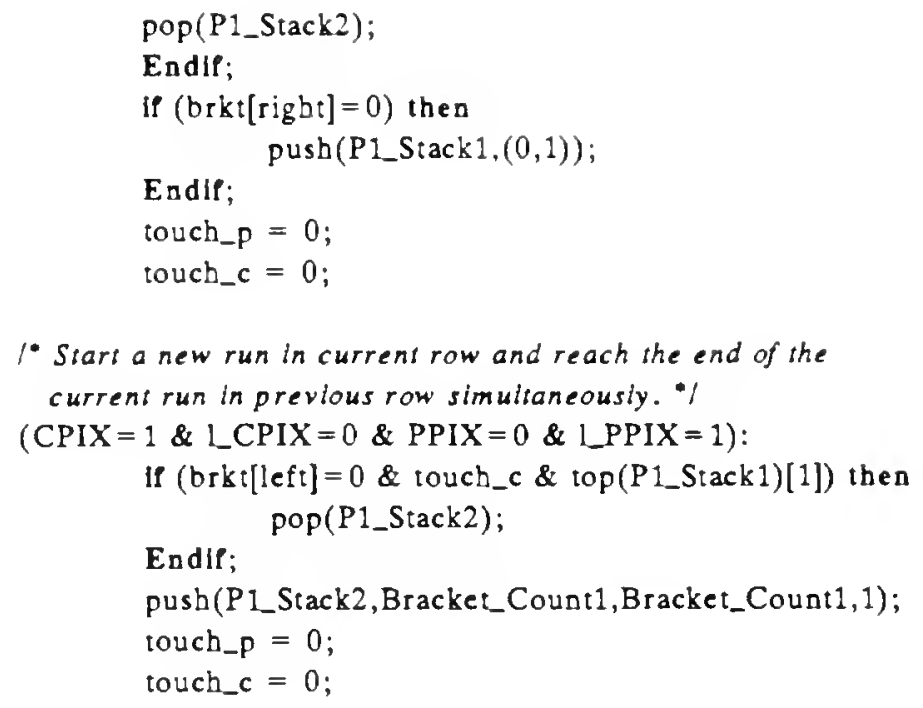

Endcase ;

Next_Pixe!_1 = true;

Endif;

1- Initialize row counters, queue couniers and set next component number to 1 , then start first sweep of row $1 \%$

Process PASS2_Initialization

If (Pass2 \& !In_Down_Load \& !In_Pass1 \& IIn_Pass2) then

In_Pass2 = true;

Row_Count $1=0$;

Row_Count2 = 511;

Queue_Count1 $=0$;

Queve_Count2 $=0$;

component_number $=1$;

Start_Row_Sweep2_of_Pass2 = true;

else

Endif;

Start_Row_Sweep2_of_Pass2 = faise;

1- Terminate pass 2 after all rows have been scanned "/

Process PASS2_Termination

If (In_Pass2 \& Next_Row_P2 \& Row_Count1 $=0$ ) then

In_Pass 2 = false;

Endif; 
1- Intilalize column counter, stacks and other varlables for the first sweep of a row in pass 2; then enable process Read_Plxel to load next plxels from current row and previous row $\because$

Process P2S1_Row_Initialization

If (Start_Pass2 | (Next_Row_P2 \& Row_Count] != 511)) then Next_Row_P2 = false;

Column_Count $=511$;

$\operatorname{reset}(\mathrm{P2}$ stack1);

$\operatorname{reset}\left(\mathrm{P}^{2} \_s t a c k 2\right)$;

$\mathrm{CPIX}=0$;

PPIX = 0;

Bracket_Count2 $=0$;

Start_Row_Sweep1_of_Pass2 = true;

else

Start_Row_Sweep1_of_Pass2 = false;

Endlf;

$1 *$ After the first sweep. from right to left, reaches the end of a row, second sweep, from left to right, is enabled *

Process End_of_Sweep1_of_Pass2

If (Next_Pixel_2 \& Column_Count $=511$ ) then

Endle;

Sweep2_of_Pass2_enable = true;

1- This is main compulation process in first sweep of a row in pass $2 \%$

Process Pixel_Computation_of_Sweep1_of_Pass2

If (P1S1_pixel_op_enable) then

P1S1_pixel_op_enable = false;

case of

1* Start a new run on both prevlous row and current row simultaneously. "

$(\mathrm{PPIX}=1 \&$ \&PPIX $=0$ \& $\mathrm{CPIX}=1 \& \&$ LCPIX $=0)$ :

case of

(brkt [right] $=0)$ :

push(P2_Stack1,(Queue(Queue_Count2),1));

(brkt right] $=1$ ):

If ! top(P2_Stack 1)[1] then

Endif;

push(P2_Stack1,(Queue(Queue_Count2),1));

Endcase;

B.9 


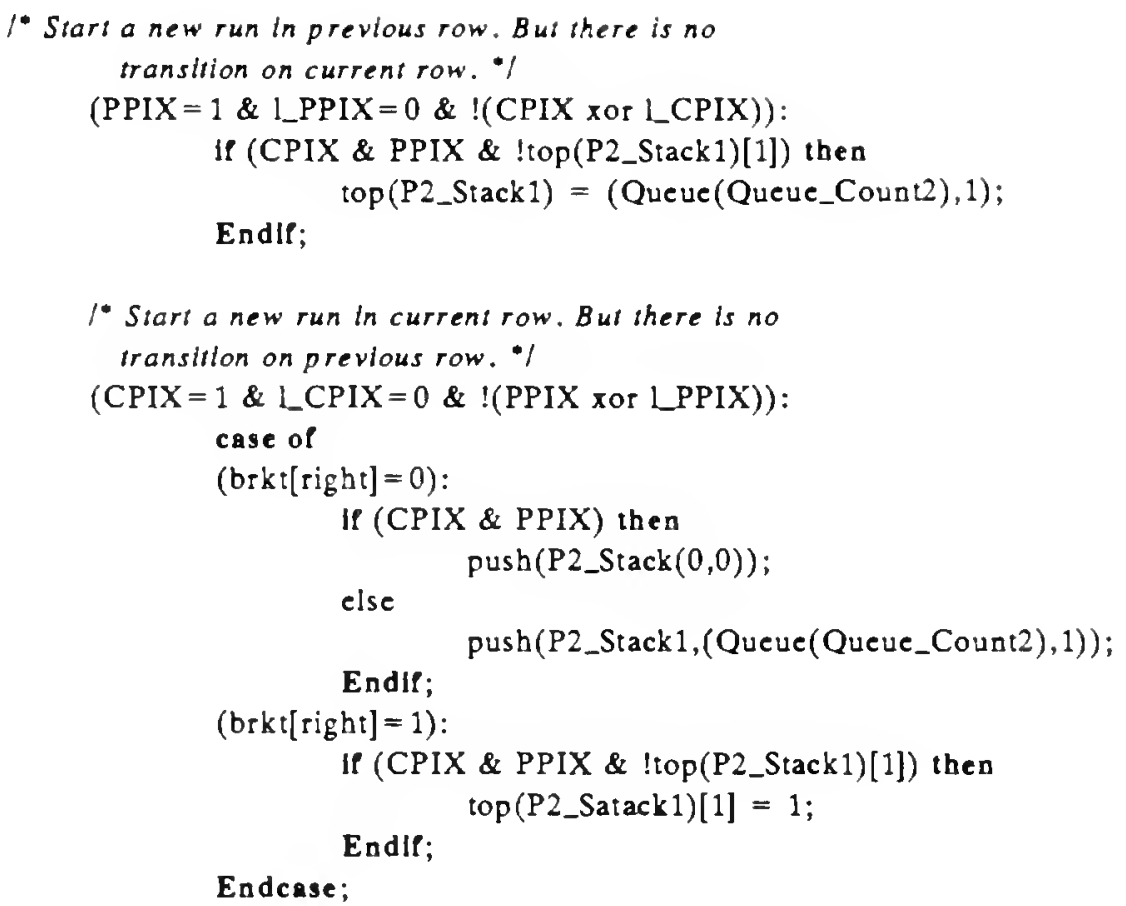

1* Reach the end of the currens run on both previous row and current row simultaneously. "I

$(\mathrm{PPIX}=0$ \& $\mathrm{LPPIX}=1$ \& $\mathrm{CPIX}=0$ \& LCPIX =1):

If (brkt[left] $=0$ ) then

EndIf; push(P2_Stack2,pop(P2_Stack1)[0]);

1* Reach the end of current run in prevlous row. But there is no transition on current row.

$(\mathrm{PPIX}=0$ \& 1_PPIX $=1 \&$ ! (CPIX xor LCPIX)): NOP;

1* Reach the end of current run in current row and there is no transition or start a new run in previous row. "

(CPIX $=0$ \& LCPIX $=1 \&$ !(PPIX xor LPPIX)):

$(\mathrm{PPIX}=1 \&$ LPPIX $=0 \&$ CPIX $=0 \&$ 1_CPIX = 1):

case of (brkt[left] $=0)$ :

If (top(P2_Stack1)[1]) then push(P2_Stack2,pop(P2_Stack1)[0]);

else push(P2_Stack2,component_number); pop(P2_Stack1); Endlf; component_number ++ ;

Endcase;

1* Start a new run in current row and reach the end of current run in prevlous row. \% 


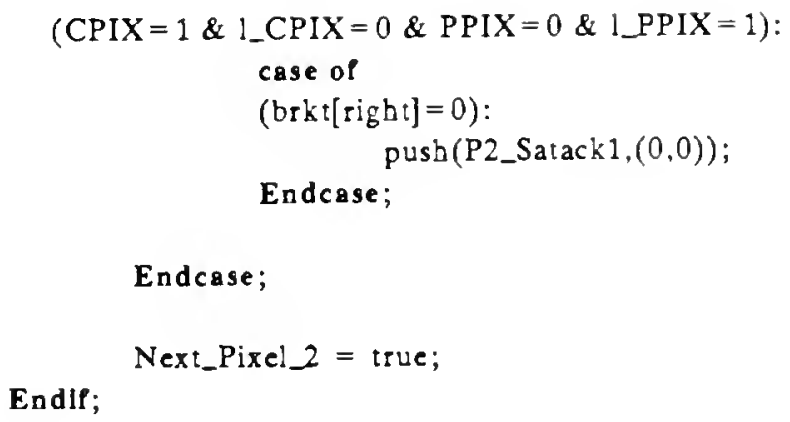

endif ;

1* After the second sweep of a row is finished, row-couniers are incremenied by one for next row. "

Process End_of_Sweep2_of_Pass2

If (Next_Pixel_3 \& Column_Count $=0)$ then

Row_Count1 + + ;

Rox_Count2 + + ;

Next_Row_P2 = true;

Endif;

1* This is main computation process in second sweep of a row in Pass $2 \%$

Process Pixel_Computation_of_Sweep2_of_Pass2

If (P1S2_pixel_op_enable) then

P1S2_pixel_op_enable = false;

case of

1* Start a new run in current row. " 


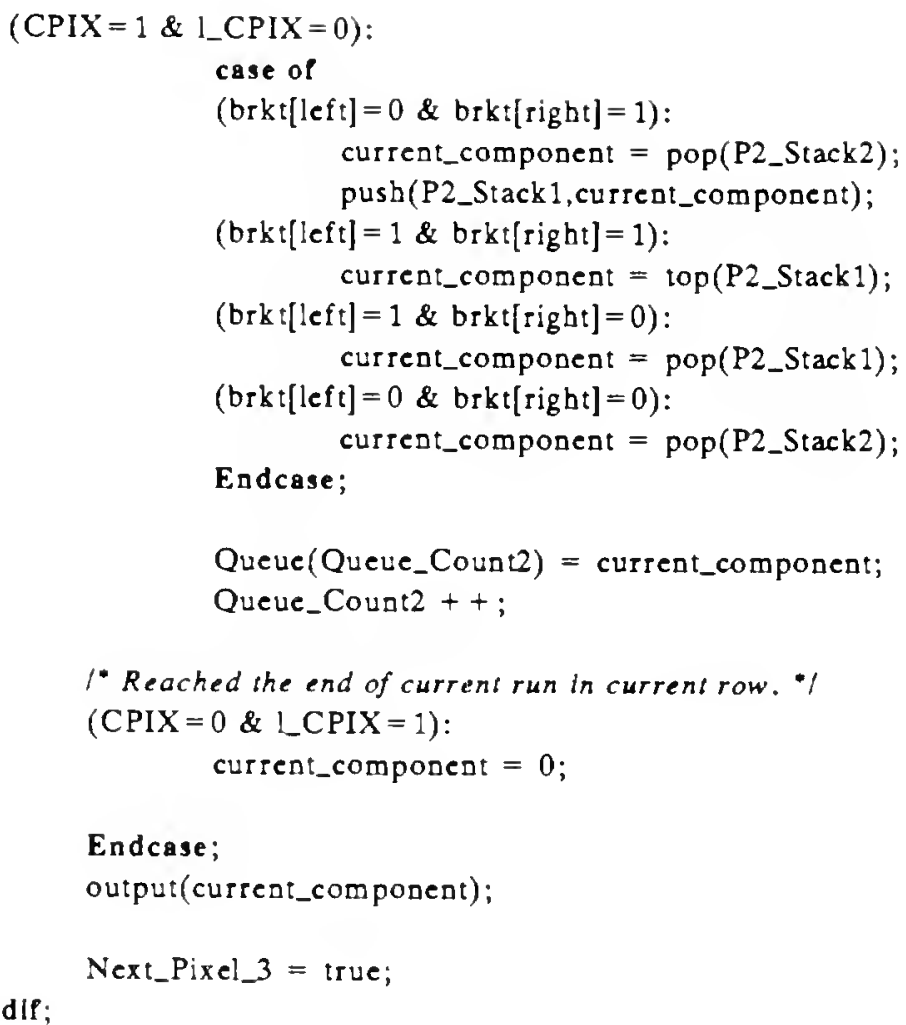



NYU COMPSCI TR-353

C. 1

Yang, Xue-Dong

Design of fast connected

components hardware.

NYU COMPSCI TR-353

C.I

Yang, Xue-Dong

Design of fast connected components hardware.

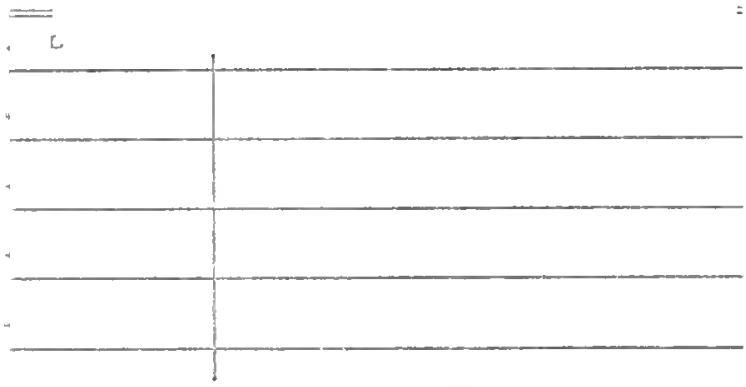

This bonk may ie kopt

1988

MOURTEEN DAYS

A fone will be charzed for each wir the bonk is kept overtim.

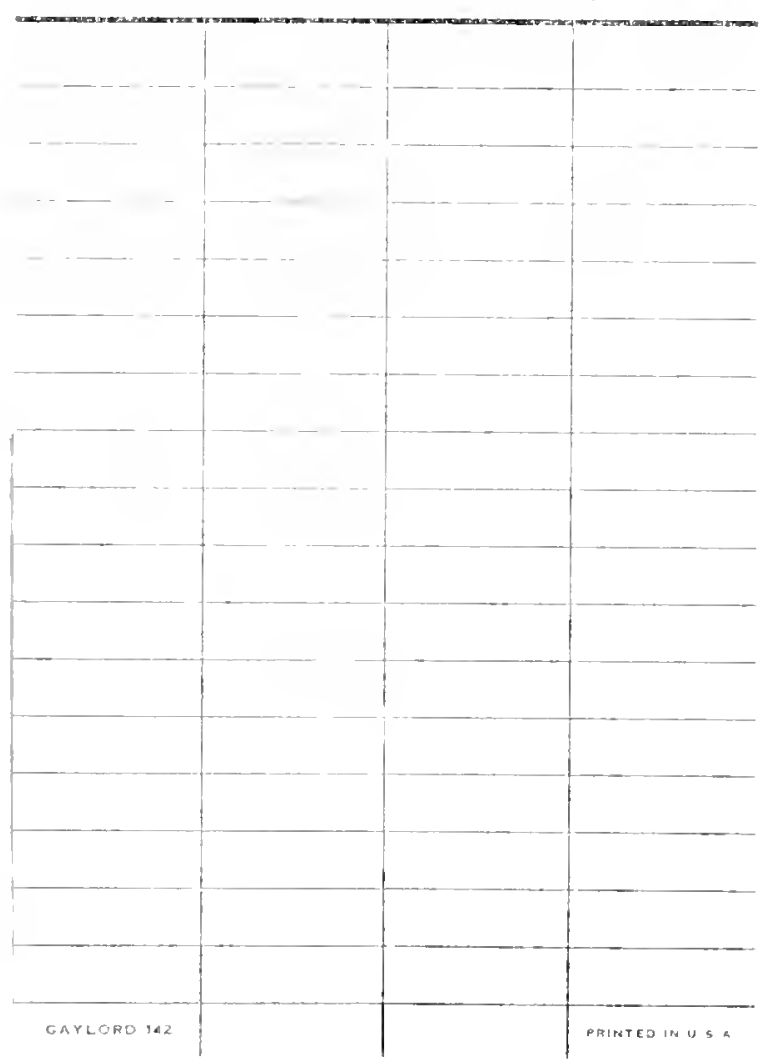


